\title{
MIXING AND DECORRELATION IN INFINITE MEASURE: THE CASE OF THE PERIODIC SINAI BILLIARD
}

\author{
FRANÇOISE PÈNE
}

\begin{abstract}
We investigate the question of the rate of mixing for observables of a $\mathbb{Z}^{d}$-extension of a probability preserving dynamical system with good spectral properties. We state general mixing results, including expansions of every order. The main part of this article is devoted to the study of mixing rate for smooth observables of the $\mathbb{Z}^{2}$-periodic Sinai billiard, with different kinds of results depending on whether the horizon is finite or infinite. We establish a first order mixing result when the horizon is infinite. In the finite horizon case, we establish an asymptotic expansion of every order, enabling the study of the mixing rate even for observables with null integrals.
\end{abstract}

\section{INTRODUCTION}

Let $(M, \nu, T)$ be a dynamical system, that is a measure space $(M, \nu)$ endowed with a measurable transformation $T: M \rightarrow M$ which preserves the measure $\nu$. The mixing properties deal with the asymptotic behaviour, as $n$ goes to infinity, of integrals of the following form

$$
C_{n}(f, g):=\int_{M} f \cdot g \circ T^{n} d \nu
$$

for suitable observables $f, g: M \rightarrow \mathbb{C}$.

Mixing properties of probability preserving dynamical systems have been studied by many authors. It is a way to measure how chaotic the dynamical system is. A probability preserving dynamical system is said to be mixing if $C_{n}(f, g)$ converges to $\int_{M} f d \nu \int_{M} g d \nu$ for every square integrable observables $f, g$. When a probability preserving system is mixing, a natural question is to study the decorrelation rate, i.e. the rate at which $C_{n}(f, g)$ converges to zero when $f$ or $g$ have null expectation. This crucial question is often a first step before proving probabilistic limit theorems (such as central limit theorem and its variants). The study of this question has a long history. Such decays of covariance have been studied for wide classes of smooth observables $f, g$ and for many probability preserving dynamical systems. In the case of the Sinai billiard, such results and further properties have been established in 26, 3, 4, 1, 2, 30, 6, 27, 28,

We are interested here in the study of mixing properties when the invariant measure $\nu$ is $\sigma$ finite. In this context, as noticed in [13], there is no satisfactory notion of mixing. Nevertheless the question of the rate of mixing for smooth observables is natural. A first step in this direction is to establish results of the following form:

$$
\lim _{n \rightarrow+\infty} \alpha_{n} C_{n}(f, g)=\int_{M} f d \nu \int_{M} g d \nu .
$$

Such results have been proved in [29, 15, 10, 15, 14] for a wide class of models and for smooth functions $f, g$, using induction on a finite measure subset of $M$.

An alternative approach, specific to the case of $\mathbb{Z}^{d}$-extensions of probability preserving dynamical system, has been pointed out in [21]. The idea therein is that, in this particular context,

Date: July 24, 2018.

2000 Mathematics Subject Classification. Primary: 37A25.

Key words and phrases. Sinai, billiard, Lorentz process, Young tower, local limit theorem, decorrelation, mixing, infinite measure. 
(11) is related to a precised local limit theorem. In the particular case of the $\mathbb{Z}^{2}$-periodic Sinai billiard with finite horizon, it has been proved in [21] that

$$
C_{n}(f, g)=\frac{c_{0}}{n} \int_{M} f d \nu \int_{M} g d \nu+o\left(n^{-1}\right),
$$

for some explicit constant $c_{0}$, for some dynamically Lipschitz functions, including functions with full support in $M$.

This paper is motivated by the question of high order expansion of mixing and by the study of the mixing rate for observables with null integrals. This last question can be seen as decorrelation rate in the infinite measure. Let us mention the fact that it has been proved in [23], for the billiard in finite horizon, that sums $\sum_{k \in \mathbb{Z}} \int_{M} f . f \circ T^{k} d \nu$ are well defined for some observables $f$ with null expectation. In the present paper, we use the approach of [21] to establish, in the context of the $\mathbb{Z}^{2}$-periodic Sinai billiard with finite horizon, a high order mixing result of the following form:

$$
C_{n}(f, g)=\sum_{m=0}^{K-1} \frac{c_{m}(f, g)}{n^{1+m}}+o\left(n^{-K}\right) .
$$

This estimate enables the study of the rate of convergence of $n C_{n}(f, g)$ to $\int_{M} f d \nu \int_{M} g d \nu$ and, most importantly, it enables the study of the rate of decay of $C_{n}(f, g)$ for functions $f$ or $g$ with integral 0 . In general, if $f$ or $g$ have zero integral we have

$$
C_{n}(f, g) \sim \frac{c_{1}(f, g)}{n^{2}}
$$

but it may happen that

$$
C_{n}(f, g) \sim \frac{c_{2}(f, g)}{n^{3}},
$$

and even that $C_{n}(f, g)=o\left(n^{-3}\right)$. For example, (2.6) gives immediately that, if $\int_{M} f d \nu \int_{M} g d \nu \neq$ 0 , then

$$
\begin{aligned}
C_{n}(f-f \circ T, g) & =C_{n}(f, g)-C_{n-1}(f, g) \\
& \sim-c_{0} \frac{\int_{M} f d \nu \cdot \int_{M} g d \nu}{n^{2}}=\frac{c_{1}(f-f \circ T, g)}{n^{2}}
\end{aligned}
$$

and

$$
\begin{aligned}
C_{n}\left(2 f-f \circ T-f \circ T^{-1}, g\right) & =C_{n}(f-f \circ T, g-g \circ T) \\
& =2 C_{n}(f, g)-C_{n-1}(f, g)-C_{n+1}(f, g) \\
& \sim-\frac{2 c_{0}}{n^{3}} \int_{M} f d \nu \int_{M} g d \nu=\frac{c_{2}(f-f \circ T, g-g \circ T)}{n^{3}} .
\end{aligned}
$$

General formulas for the dominating term will be given in Theorem 4.5, Remark 4.6 and Corollary 4.7. In particular $c_{1}(f, g)$ and $c_{2}(f, g)$ will be precised.

We point out the fact that the method we use is rather general in the context of $\mathbb{Z}^{d}$-extensions over dynamical systems with good spectral properties, and that, to our knowledge, these are the first results of this kind for dynamical systems preserving an infinite measure.

We establish moreover an estimate of the following form for smooth observables of the $\mathbb{Z}^{2}$ periodic Sinai billiard with infinite horizon:

$$
C_{n}(f, g)=\frac{c_{0}}{n \log n} \int_{M} f d \nu \int_{M} g d \nu+o\left((n \log n)^{-1}\right) .
$$

The paper is organized as follows. In Section 1, we present the model of the $\mathbb{Z}^{2}$-periodic Sinai billiard and we state our main results for this model (finite/infinite horizon). In Section 2, we state general mixing results for $\mathbb{Z}^{d}$-extensions of probability preserving dynamical systems for 
which the Nagaev-Guivarc'h perturbation method can be implemented. In Section 3, we recall some facts on the towers constructed by Young for the Sinai billiards. In Section 4, we prove our main results for the billiard in finite horizon (see also Appendix A for the computation of the first coefficients). In Section 5, we prove our result for the billiard in infinite horizon.

\section{MAin Results For $\mathbb{Z}^{2}$-PERIOdiC Sinai billiards}

Let us introduce the $\mathbb{Z}^{2}$-periodic Sinai billiard $(M, \nu, T)$.

Billiards systems modelise the behaviour of a point particle moving at unit speed in a domain $Q$ and bouncing off $\partial Q$ with respect to the Descartes reflection law (incident angle=reflected angle). We assume here that $Q:=\mathbb{R}^{2} \backslash \bigcup_{\ell \in \mathbb{Z}^{2}} \bigcup_{i=1}^{I}\left(O_{i}+\ell\right)$, with $I \geq 2$ and where $O_{1}, \ldots, O_{I}$ are convex bounded open sets (the boundaries of which are $C^{3}$-smooth and have non null curvature). We assume that the closures of the obstacles $O_{i}+\ell$ are pairwise disjoint. The billiard is said to have finite horizon if every line in $\mathbb{R}^{2}$ meets $\partial Q$. Otherwise it is said to have infinite horizon.

We consider the dynamical system $(M, \nu, T)$ corresponding to the dynamics at reflection times which is defined as follows. Let $M$ be the set of reflected vectors off $\partial Q$, i.e.

$$
M:=\left\{(q, \vec{v}) \in \partial Q \times S^{1}:\langle\vec{n}(q), \vec{v}\rangle \geq 0\right\},
$$

where $\vec{n}(q)$ stands for the unit normal vector to $\partial Q$ at $q$ directed inward $Q$. We decompose this set into $M:=\bigcup_{\ell \in \mathbb{Z}^{2}} \mathcal{C}_{\ell}$, with

$$
\mathcal{C}_{\ell}:=\left\{(q, \vec{v}) \in M: q \in \bigcup_{i=1}^{I}\left(\partial O_{i}+\ell\right)\right\} .
$$

The set $\mathcal{C}_{\ell}$ is called the $\ell$-cell. We define $T: M \rightarrow M$ as the transformation mapping a reflected vector at a reflection time to the reflected vector at the next reflection time. We consider the measure $\nu$ absolutely continuous with respect to the Lebesgue measure on $M$, with density proportional to $(q, \vec{v}) \mapsto\langle\vec{n}(q), \vec{v}\rangle$ and such that $\nu\left(\mathcal{C}_{0}\right)=1$.

Because of the $\mathbb{Z}^{2}$-periodicity of the model, there exists a transformation $\bar{T}: \mathcal{C}_{0} \rightarrow \mathcal{C}_{0}$ and a function $\kappa: \mathcal{C}_{0} \rightarrow \mathbb{Z}^{2}$ such that

$$
\forall((q, \vec{v}), \ell) \in \mathcal{C}_{0} \times \mathbb{Z}^{2}, T(q+\ell, \vec{v})=\left(q^{\prime}+\ell+\kappa(q, \vec{v}), \vec{v}^{\prime}\right), \text { if } \bar{T}(q, \vec{v})=\left(q^{\prime}, \vec{v}^{\prime}\right) .
$$

This allows us to define a probability preserving dynamical $(\bar{M}, \bar{\mu}, \bar{T})$ (the Sinai billiard) by setting $\bar{M}:=\mathcal{C}_{0}$ and $\bar{\mu}=\nu_{\mid \mathcal{C}_{0}}$. Note that (4) means that $(M, \nu, T)$ can be represented by the $\mathbb{Z}^{2}$-extension of $(\bar{M}, \bar{\mu}, \bar{T})$ by $\kappa$. In particular, iterating (4) leads to

$$
\forall((q, \vec{v}), \ell) \in \mathcal{C}_{0} \times \mathbb{Z}^{2}, T^{n}(q+\ell, \vec{v})=\left(q_{n}^{\prime}+\ell+S_{n}(q, \vec{v}), \vec{v}_{n}^{\prime}\right),
$$

if $\bar{T}^{n}(q, \vec{v})=\left(q_{n}^{\prime}, \vec{v}_{n}^{\prime}\right)$ and with the notation

$$
S_{n}:=\sum_{k=0}^{n-1} \kappa \circ \bar{T}^{k} .
$$

The set of tangent reflected vectors $\mathcal{S}_{0}$ given by

$$
\mathcal{S}_{0}:=\{(q, \vec{v}) \in M:\langle\vec{v}, \vec{n}(q)\rangle=0\}
$$

plays a special role in the study of $T$. Note that $T$ defines a $C^{1}$-diffeomorphism from $M \backslash\left(\mathcal{S}_{0} \cup\right.$ $\left.T^{-1}\left(\mathcal{S}_{0}\right)\right)$ to $M \backslash\left(\mathcal{S}_{0} \cup T\left(\mathcal{S}_{0}\right)\right)$.

Statistical properties of $(\bar{M}, \bar{\mu}, \bar{T})$ have been studied by many authors since the seminal article [26] by Sinai.

In the finite horizon case, limit theorems have been established in [4, 2, 30, 6], including the convergence in distribution of $\left(S_{n} / \sqrt{n}\right)_{n}$ to a centered gaussian random variable $B$ with 
nondegenerate variance matrix $\Sigma^{2}$ given by:

$$
\Sigma^{2}:=\sum_{k \in \mathbb{Z}} \mathbb{E}_{\bar{\mu}}\left[\kappa \otimes \kappa \circ \bar{T}^{k}\right]
$$

where we used the notation $X \otimes Y$ for the matrix $\left(x_{i} y_{j}\right)_{i, j}$, for $X=\left(x_{i}\right)_{i}, Y=\left(y_{j}\right)_{j} \in \mathbb{C}^{2}$. Moreover a local limit theorem for $S_{n}$ has been established in [27] and some of its refinements have been stated and used in [9, 19, 20, 22] with various applications. Recurrence and ergodicity of this model follow from [8, 24, 27, 25, 18].

In the infinite horizon case, a result of exponential decay of correlation has been proved in [6]. A nonstandard central limit theorem (with normalization in $\sqrt{n \log n}$ ) and a local limit theorem have been established in [28], ensuring recurrence and ergodicity of the infinite measure system $(M, \nu, T)$. This result states in particular that $\left(S_{n} / \sqrt{n \log n}\right)_{n}$ converges in distribution to a centered gaussian distribution with variance $\Sigma_{\infty}^{2}$ given by

$$
\Sigma_{\infty}^{2}:=\sum_{x \in \mathcal{S}_{0} \mid \bar{T} x=x} \frac{d_{x}^{2}}{2|\kappa(x)| \sum_{i=1}^{I}\left|\partial O_{i}\right|}(\kappa(x))^{\otimes 2},
$$

where $d_{x}$ is the width of the corridor corresponding to $x$.

Our main results provide mixing estimates for dynamically Lipschitz functions. Let us introduce this class of observables. Let $\xi \in(0,1)$. We consider the metric $d_{\xi}$ on $M$ given by

$$
\forall x, y \in M, \quad d_{\xi}(x, y):=\xi^{s(x, y)},
$$

where $s$ is a separation time defined as follows: $s(x, y)$ is the maximum of the integers $k \geq 0$ such that $x$ and $y$ lie in the same connected component of $M \backslash \bigcup_{j=-k}^{k} T^{-j} \mathcal{S}_{0}$. For every $f: M \rightarrow \mathbb{C}$, we write $L_{\xi}(f)$ for the Lipschitz constant with respect to $d_{\xi}$ :

$$
L_{\xi}(f):=\sup _{x \neq y} \frac{|f(x)-f(y)|}{d_{\xi}(x, y)} .
$$

We then set

$$
\|f\|_{(\xi)}:=\|f\|_{\infty}+L_{\xi}(f) .
$$

Before stating our main result, let us introduce some additional notations.

We will work with symmetric multilinear forms. For any $A=\left(A_{i_{1}, \ldots, i_{m}}\right)_{\left(i_{1}, \ldots, i_{m}\right) \in\{1,2\}^{m}}$ and $B=\left(B_{i_{1}, \ldots, i_{k}}\right)_{\left(i_{1}, \ldots, i_{k}\right) \in\{1,2\}^{k}}$ with complex entries $(A$ and $B$ are identified respectively with a $m$-multilinear form on $\mathbb{C}^{2}$ and with a $k$-multilinear form on $\mathbb{C}^{2}$ ), we define $A \otimes B$ as the element $C$ of $\mathbb{C}^{\{1,2\}^{m+m^{\prime}}}$ (identified with a $\left(m+m^{\prime}\right)$-multilinear form on $\left.\mathbb{C}^{2}\right)$ such that

$$
\forall i_{1},, \ldots, i_{m+m^{\prime}} \in\{1,2\}, \quad C_{\left(i_{1}, \ldots, i_{m+m^{\prime}}\right)}=A_{\left(i_{1}, \ldots, i_{m}\right)} B_{\left(i_{m+1} \ldots, i_{m+m^{\prime}}\right)} .
$$

For any $A=\left(A_{i_{1}, \ldots, i_{m}}\right)_{\left(i_{1}, \ldots, i_{m}\right) \in\{1,2\}^{m}}$ and $B=\left(B_{i_{1}, \ldots, i_{k}}\right)_{\left(i_{1}, \ldots, i_{k}\right) \in\{1,2\}^{k}}$ symmetric with complex entries with $k \leq m$, we define $A * B$ as the element $C$ of $\mathbb{C}^{\{1,2\}^{m-k}}$ (identified with a $(m-k)$ multilinear form on $\mathbb{C}^{2}$ ) such that

$$
\forall i_{1},, \ldots, i_{m-k} \in\{1,2\}, \quad C_{\left(i_{1}, \ldots, i_{m-k}\right)}=\sum_{i_{m-k+1}, \ldots, i_{m} \in\{1,2\}} A_{\left(i_{1}, \ldots, i_{m}\right)} B_{\left(i_{m-k+1}, \ldots, i_{m}\right)} .
$$

We identify naturally vectors in $\mathbb{C}^{2}$ with 1-linear functions and symmetric matrices with symmetric bilinear functions. For any $C^{m}$-smooth function $F: \mathbb{C}^{2} \rightarrow \mathbb{C}$, we write $F^{(m)}$ for its $m$-th differential, which is identified with a $m$-linear function on $\mathbb{C}^{2}$. We write $A^{\otimes k}$ for the product $A \otimes \ldots \otimes A$. Observe that, with these notations, Taylor expansions of $F$ at 0 are simply written

$$
\sum_{k=0}^{m} F^{(k)}(0) * x^{\otimes k}
$$


It is also worth noting that $A *(B \otimes C)=(A * B) * C$, for every $A, B, C$ corresponding to symmetric multilinear forms with respective ranks $m, k, \ell$ with $m \geq k+\ell$.

We extend the definition of $\kappa$ to $M$ by setting $\kappa((q+\ell, \vec{v}))=\kappa(q, \vec{v})$ for every $(q, \vec{v}) \in \bar{M}$ and every $\ell \in \mathbb{Z}^{2}$. For every $k \in \mathbb{Z}$ and every $x \in M$, we write $\mathcal{I}_{k}(x)$ for the label in $\mathbb{Z}^{2}$ of the cell containing $T^{k} x$, i.e. $\mathcal{I}_{k}$ is the label of the cell in which the particle is at the $k$-th reflection time. It is worth noting that, for $n \geq 0$, we have $\mathcal{I}_{n}-\mathcal{I}_{0}=\sum_{k=0}^{n-1} \kappa \circ T^{k}$ and $\mathcal{I}_{-n}-\mathcal{I}_{0}=-\sum_{k=-n}^{-1} \kappa \circ T^{k}$.

Now let us state our main results, the proofs of which are postponed to Section 4, We start by stating our result in the infinite horizon case, and then we will present sharper results in the finite horizon case.

\section{1. $\mathbb{Z}^{2}$-periodic Sinai billiard with infinite horizon.}

Theorem 1.1. Let $(M, \nu, T)$ be the $\mathbb{Z}^{2}$-periodic Sinai billiard with infinite horizon. Suppose that the set of corridor free flights $\left\{\kappa(x), x \in \mathcal{S}_{0}, \bar{T} x=x\right\}$ spans $\mathbb{R}^{2}$. Let $f, g: M \rightarrow \mathbb{C}$ (with respect to $d_{\xi}$ ) be two dynamically Lipschitz continuous functions such that

$$
\sum_{\ell \in \mathbb{Z}^{2}}\left(\left\|f \mathbf{1}_{\mathcal{C}_{\ell}}\right\|_{\infty}+\left\|g \mathbf{1}_{\mathcal{C}_{\ell}}\right\|_{\infty}\right)<\infty
$$

Then

$$
\int_{M} f . g \circ T^{n} d \nu=\frac{1}{2 \pi \sqrt{\operatorname{det} \Sigma_{\infty}^{2}} n \log n}\left(\int_{M} f d \nu \int_{M} g d \nu+o(1)\right) .
$$

1.2. $\mathbb{Z}^{2}$-periodic Sinai billiard with finite horizon. We first state our result providing an expansion of every order for the mixing (see Theorem 4.5 and Corollary 4.7 for more details).

Theorem 1.2. Let $K$ be a positive integer. Let $f, g: M \rightarrow \mathbb{C}$ be two dynamically Lipschitz continuous observables such that

$$
\sum_{\ell \in \mathbb{Z}^{2}}|\ell|^{2 K-2}\left(\left\|f \mathbf{1}_{\mathcal{C}_{\ell}}\right\|_{(\xi)}+\left\|g \mathbf{1}_{\mathcal{C}_{\ell}}\right\|_{(\xi)}\right)<\infty
$$

then there exist $c_{0}(f, g), \ldots, c_{K-1}(f, g)$ such that

$$
\int_{M} f . g \circ T^{n} d \nu=\sum_{m=0}^{K-1} \frac{c_{m}(f, g)}{n^{1+m}}+o\left(n^{-K}\right) .
$$

We precise in the following theorem the expansion of order 2.

Theorem 1.3. Let $f, g: M \rightarrow \mathbb{R}$ be two bounded observables such that

$$
\sum_{\ell \in \mathbb{Z}^{2}}|\ell|^{2}\left(\left\|f \mathbf{1}_{\mathcal{C}_{\ell}}\right\|_{(\xi)}+\left\|g \mathbf{1}_{\mathcal{C}_{\ell}}\right\|_{(\xi)}\right)<\infty
$$

Then

$$
\begin{aligned}
\int_{M} f . g \circ T^{n} d \nu= & \frac{1}{2 \pi \sqrt{\operatorname{det} \Sigma^{2}}}\left\{\frac{1}{n} \int_{M} f d \nu \int_{M} g d \nu+\frac{1}{2 n^{2}} \Sigma^{-2} * \tilde{\mathfrak{A}}_{2}(f, g)\right. \\
& \left.+\frac{1}{4 ! n^{2}} \int_{M} f d \nu \int_{M} g d \nu\left(\Sigma^{-2}\right)^{\otimes 2} * \Lambda_{4}\right\}+o\left(n^{-2}\right)
\end{aligned}
$$

with $\Sigma^{-2}=\left(\Sigma^{2}\right)^{-1}$ and

$$
\begin{gathered}
\tilde{\mathfrak{A}}_{2}(f, g):=-\int_{M} f d \nu \mathfrak{B}_{2}^{-}(g)-\int_{M} g d \nu \mathfrak{B}_{2}^{+}(f)-\int_{M} f d \nu \int_{M} g d \nu \mathfrak{B}_{0}+2 \mathfrak{B}_{1}^{+}(f) \otimes \mathfrak{B}_{1}^{-}(g), \\
\mathfrak{B}_{2}^{+}(f):=\lim _{m \rightarrow+\infty} \int_{M} f \cdot\left(\mathcal{I}_{m}^{\otimes 2}-m \Sigma^{2}\right) d \nu,
\end{gathered}
$$




$$
\begin{gathered}
\mathfrak{B}_{2}^{-}(g):=\lim _{m \rightarrow-\infty} \int_{M} g \cdot\left(\mathcal{I}_{m}^{\otimes 2}-|m| \Sigma^{2}\right) d \nu \\
\mathfrak{B}_{1}^{+}(f):=\lim _{m \rightarrow+\infty} \int_{M} f \cdot \mathcal{I}_{m} d \nu, \quad \mathfrak{B}_{1}^{-}(g):=\lim _{m \rightarrow-\infty} \int_{M} g \cdot \mathcal{I}_{m} d \nu, \\
\mathfrak{B}_{0}=\lim _{m \rightarrow+\infty}\left(m \Sigma^{2}-\mathbb{E}_{\bar{\mu}}\left[S_{m}^{\otimes 2}\right]\right)
\end{gathered}
$$

and

$$
\Lambda_{4}:=\lim _{n \rightarrow+\infty} \frac{\mathbb{E}_{\bar{\mu}}\left[S_{n}^{\otimes 4}\right]-3 n^{2}\left(\Sigma^{2}\right)^{\otimes 2}}{n}+6 \Sigma^{2} \otimes \mathfrak{B}_{0} .
$$

Observe that we recover (3) since $\Sigma^{2} * \Sigma^{-2}=2$,

$$
\mathfrak{B}_{1}^{+}(f-f \circ T)=\lim _{m \rightarrow+\infty} \int_{M} f . \kappa \circ T^{m} d \nu=0
$$

and

$$
\begin{aligned}
\mathfrak{B}_{2}^{+}(f-f \circ T) & =\lim _{n \rightarrow+\infty} \int_{M} f \cdot\left(\mathcal{I}_{m}^{\otimes 2}-\mathcal{I}_{m-1}^{\otimes 2}\right) \\
& =\lim _{m \rightarrow+\infty} \int_{M} f \cdot\left(\kappa^{\otimes 2} \circ T^{m-1}+2 \sum_{k=0}^{m-2}\left(\kappa \circ T^{k}\right) \otimes \kappa \circ T^{m-1}\right) d \nu \\
& =\lim _{m \rightarrow+\infty} \int_{M} f d \nu \mathbb{E}_{\bar{\mu}}\left[\kappa^{\otimes 2}+2 \sum_{k=1}^{m-1} \kappa \otimes \kappa \circ T^{k}\right], \\
& =\Sigma^{2} \int_{M} f d \nu,
\end{aligned}
$$

where we used Proposition A.1.

Remark 1.4. Note that

$$
\begin{aligned}
\mathfrak{B}_{2}^{+}(f)= & \sum_{j, m \geq 0} \int_{M} f \cdot\left(\kappa \circ T^{j} \otimes \kappa \circ T^{m}-\mathbb{E}_{\bar{\mu}}\left[\kappa \circ \bar{T}^{j} \otimes \kappa \circ \bar{T}^{m}\right]\right) d \nu \\
& +\int_{M} f \mathcal{I}_{0}^{\otimes 2} d \nu+2 \sum_{m \geq 0} \int_{M} f \cdot \mathcal{I}_{0} \otimes \kappa \circ \bar{T}^{m} d \nu-\mathfrak{B}_{0} \int_{M} f d \nu \\
\mathfrak{B}_{2}^{-}(g)= & \sum_{j, m \leq-1} \int_{M} g \cdot\left(\kappa \circ T^{j} \otimes \kappa \circ T^{m}-\mathbb{E}_{\bar{\mu}}\left[\kappa \circ \bar{T}^{j} \otimes \kappa \circ \bar{T}^{m}\right]\right) d \nu \\
& +\int_{M} g \cdot \mathcal{I}_{0}^{\otimes 2} d \nu-2 \sum_{m \leq-1} \int_{M} g \cdot \mathcal{I}_{0} \otimes \kappa \circ T^{m} d \nu-\mathfrak{B}_{0} \int_{M} g d \nu, \\
& \mathfrak{B}_{1}^{+}(f)=\sum_{m \geq 0} \int_{M} f \cdot \kappa \circ T^{m} d \nu+\int_{M} f \cdot \mathcal{I}_{0} d \nu, \\
& \mathfrak{B}_{1}^{-}(g)=-\sum_{m \leq-1} \int_{M} g \cdot \kappa \circ T^{m} d \nu+\int_{M} g \cdot \mathcal{I}_{0} d \nu
\end{aligned}
$$

and

$$
\mathfrak{B}_{0}=\sum_{m \in \mathbb{Z}}|m| \mathbb{E}_{\bar{\mu}}\left[\kappa \otimes \kappa \circ \bar{T}^{m}\right] .
$$

Corollary 1.5. Under the assumptions of Theorem 1.3, if $\int_{M} f d \nu=0$ and $\int_{M} g d \nu=0$, then

$$
\int_{M} f . g \circ T^{n} d \nu=\frac{\Sigma^{-2} *\left(\mathfrak{B}_{1}^{+}(f) \otimes \mathfrak{B}_{1}^{-}(g)\right)}{n^{2} 2 \pi \sqrt{\operatorname{det} \Sigma^{2}}}+o\left(n^{-2}\right) .
$$


Two natural examples of zero integral functions are $\mathbf{1}_{\mathcal{C}_{0}}-\mathbf{1}_{\mathcal{C}_{e_{1}}}$ with $e_{1}=(1,0)$ or $f \mathcal{C}_{0}$ with $\int_{\mathcal{C}_{0}} f d \nu=0$. Note that

$$
\int_{M}\left(\left(\mathbf{1}_{\mathcal{C}_{0}}-\mathbf{1}_{\mathcal{C}_{e_{1}}}\right) \cdot\left(\mathbf{1}_{\mathcal{C}_{0}}-\mathbf{1}_{\mathcal{C}_{e_{1}}}\right) \circ T^{n}\right) d \nu \sim \frac{\sigma_{2,2}^{2}}{n^{2} 2 \pi\left(\operatorname{det} \Sigma^{2}\right)^{3 / 2}},
$$

with $\Sigma^{2}=\left(\sigma_{i, j}^{2}\right)_{i, j=1,2}$ and that

$$
\int_{M}\left(f \mathbf{1}_{\mathcal{C}_{0}} \cdot \mathbf{1}_{\mathcal{C}_{0}} \circ T^{n}\right) d \nu \sim-\frac{1}{n^{2} 2 \pi\left(\operatorname{det} \Sigma^{2}\right)^{3 / 2}} \sum_{m \geq 0} \mathbb{E}_{\bar{\mu}}\left[f \cdot\left(\sigma_{2,2}^{2} \kappa_{1}+\sigma_{1,1}^{2} \kappa_{2}\right) \circ T^{m}\right],
$$

with $\kappa=\left(\kappa_{1}, \kappa_{2}\right)$, provided the sum appearing in the last formula is non null. As noticed in introduction, it may happen that (77) provides only $\int_{M} f . g \circ T^{n}=o\left(n^{-2}\right)$. This is the case for example if $\int_{M} g d \nu=0$ and if $f$ has the form $f(q+\ell, \vec{v})=f_{0}(q, \vec{v}) \cdot h_{\ell}$ with $\mathbb{E}_{\bar{\mu}}\left[f_{0}\right]=0$ and $\sum_{\ell} h_{\ell}=0$.

Hence it can be useful to go further in the asymptotic expansion, which is possible thanks to Theorem 4.5. A formula for the term of order $n^{-3}$ when $\int_{M} f d \nu=\int_{M} g d \nu=\tilde{\mathfrak{A}}_{2}(f, g)=0$ is stated in theorem 4.8 and gives the following estimate, showing that, for some observables, $C_{n}(f, g)$ has order $n^{-3}$.

Proposition 1.6. If $f$ and $g$ can be decomposed in $f(q+\ell, \vec{v})=f_{0}(q, \vec{v}) \cdot h_{\ell}$ and $g(q+\ell, \vec{v})=$ $g_{0}(q, \vec{v}) . q_{\ell}$ with $\mathbb{E}_{\bar{\mu}}\left[f_{0}\right]=\mathbb{E}_{\bar{\mu}}\left[g_{0}\right]=0$ and $\sum_{\ell} q_{\ell}=\sum_{\ell} h_{\ell}=0$ such that $\sum_{\ell \in \mathbb{Z}^{2}}|\ell|^{4}\left(\left\|f \mathbf{1}_{\mathcal{C}_{\ell}}\right\|_{(\xi)}+\right.$ $\left.\left\|g \mathbf{1}_{\mathcal{C}_{\ell}}\right\|_{(\xi)}\right)<\infty$. Then

$$
\int_{M} f . g \circ T^{n} d \nu=\frac{\left(\Sigma^{-2}\right)^{\otimes 2}}{2 \pi \sqrt{\operatorname{det} \Sigma^{2}} n^{3}} * \frac{\mathfrak{B}_{2}^{+}(f) \otimes \mathfrak{B}_{2}^{-}(g)}{4}+o\left(n^{-3}\right),
$$

with here

$\frac{\mathfrak{B}_{2}^{+}(f) \otimes \mathfrak{B}_{2}^{-}(g)}{4}=-\left(\sum_{\ell \in \mathbb{Z}^{2}} h_{\ell} \cdot \ell\right) \otimes\left(\sum_{j \geq 0} \mathbb{E}_{\bar{\mu}}\left[f_{0} . \kappa \circ T^{j}\right]\right) \otimes\left(\sum_{\ell \in \mathbb{Z}^{2}} q_{\ell \cdot \ell}\right) \otimes\left(\sum_{m \leq-1} \mathbb{E}_{\bar{\mu}}\left[g_{0} . \kappa \circ T^{m}\right]\right)$.

\section{General Results for $\mathbb{Z}^{d}$-EXtensions And Key iDEAS}

In this section we state general results in the general context of $\mathbb{Z}^{d}$-extensions over dynamical systems satisfying good spectral properties. This section contains the rough ideas of the proofs for the billiard, without some complications due to the quotient tower. Moreover the generality of our assumptions makes our results implementable to a wide class of models with present and future developments of the Nagaev-Guivarch method of perturbation of transfer operators.

We consider a dynamical system $(M, \nu, T)$ given by the $\mathbb{Z}^{d}$-extension of a probability preserving dynamical system $(\bar{M}, \bar{\mu}, \bar{T})$ by $\kappa: \bar{M} \rightarrow \mathbb{Z}^{d}$. This means that $M=\bar{M} \times \mathbb{Z}^{d}, \nu=\bar{\mu} \otimes \mathfrak{m}_{d}$ where $\mathfrak{m}_{d}$ is the counting measure on $\mathbb{Z}^{d}$ and with

$$
\forall(x, \ell) \in \bar{M} \times \mathbb{Z}^{d}, \quad T(x, \ell)=(\bar{T}(x), \ell+\kappa(x)),
$$

so that

$$
\forall(x, \ell) \in \bar{M} \times \mathbb{Z}^{d}, \forall n \geq 1, \quad T^{n}(x, \ell)=\left(\bar{T}^{n}(x), \ell+S_{n}(x)\right),
$$

with $S_{n}:=\sum_{k=0}^{n-1} \kappa \circ \bar{T}^{k}$. Let $P$ be the transfer operator of $\bar{T}$, i.e. the dual operator of $f \mapsto f \circ \bar{T}$. Our method is based on the following key fomulas:

$$
\begin{aligned}
\int_{M} f . g \circ T^{n} d \nu & =\sum_{\ell, \ell^{\prime} \in \mathbb{Z}^{2}} \mathbb{E}_{\bar{\mu}}\left[f(\cdot, \ell) . \mathbf{1}_{\left.S_{n}=\ell^{\prime}-\ell \cdot g\left(\bar{T}^{n}(\cdot), \ell^{\prime}\right)\right]}\right. \\
& =\sum_{\ell, \ell^{\prime} \in \mathbb{Z}^{d}} \mathbb{E}_{\bar{\mu}}\left[P^{n}\left(\mathbf{1}_{S_{n}=\ell^{\prime}-\ell} f(\cdot, \ell)\right) g\left(\cdot, \ell^{\prime}\right)\right]
\end{aligned}
$$


and

$$
\begin{aligned}
P^{n}\left(\mathbf{1}_{S_{n}=\ell} u\right) & =\frac{1}{(2 \pi)^{d}} \int_{[-\pi, \pi]^{d}} e^{-i t * \ell} P^{n}\left(e^{i t * S_{n}} u\right) d t \\
& =\frac{1}{(2 \pi)^{d}} \int_{[-\pi, \pi]^{d}} e^{-i t * \ell} P_{t}^{n}(u) d t,
\end{aligned}
$$

with $P_{t}:=P\left(e^{i t * \kappa} \cdot\right)$. Note that (9) makes a link between mixing properties and the local limit theorem and that (10) shows the importance of the study of the family of perturbed operators $\left(P_{t}\right)_{t}$ in this study.

We will make the following general assumptions about $\left(P_{t}\right)_{t}$.

Hypothesis 2.1 (Spectral hypotheses). There exist two complex Banach spaces $(\mathcal{B},\|\cdot\|)$ and $\left(\mathcal{B}_{0},\|\cdot\|_{0}\right)$ such that:

- $\mathcal{B} \hookrightarrow \mathcal{B}_{0} \hookrightarrow L^{1}(\bar{M}, \bar{\mu})$ and $\mathbf{1}_{\bar{M}} \in \mathcal{B}$,

- there exist constants $b \in(0, \pi], C>0$ and $\vartheta \in(0,1)$ and three functions $\lambda .:[-b, b]^{d} \rightarrow \mathbb{C}$ and $\Pi$., $R .:[-b, b]^{d} \rightarrow \mathcal{L}(\mathcal{B}, \mathcal{B})$ such that $\lim _{t \rightarrow 0} \lambda_{t}=1$ and $\lim _{t \rightarrow 0}\left\|\Pi_{t}-\mathbb{E}_{\mu}[\cdot] \mathbf{1}_{\bar{M}}\right\|_{\mathcal{L}\left(\mathcal{B}, \mathcal{B}_{0}\right)}=$ 0 and such that, in $\mathcal{L}(\mathcal{B}, \mathcal{B})$,

$$
\begin{gathered}
\forall u \in[-b, b]^{d}, \quad P_{u}=\lambda_{u} \Pi_{u}+R_{u}, \quad \Pi_{u} R_{u}=R_{u} \Pi_{u}=0, \quad \Pi_{u}^{2}=\Pi_{u}, \\
\sup _{u \in[-b, b]^{d}}\left\|R_{u}^{k}\right\|_{\mathcal{L}\left(\mathcal{B}, \mathcal{B}_{0}\right)} \leq C \vartheta^{k}, \quad \sup _{u \in[-\pi, \pi]^{d} \backslash[-b, b]^{d}}\left\|P_{u}^{k}\right\|_{\mathcal{L}\left(\mathcal{B}, \mathcal{B}_{0}\right)} \leq C \vartheta^{k} .
\end{gathered}
$$

Note that (11) ensures that

$$
\forall u \in[-b, b], \quad P_{u}^{n}=\lambda_{u}^{n} \Pi_{u}+R_{u}^{n} .
$$

We will make the following assumption on the expansion of $\lambda$ at 0 .

Hypothesis 2.2. Let $Y$ be a random variable with integrable characteristic function $a .:=e^{-\psi(\cdot)}$ and with density function $\Phi$. Assume that there exists a sequence of invertible matrices $\left(\Theta_{n}\right)_{n}$ such that $\lim _{n \rightarrow+\infty} \Theta_{n}^{-1}=0$ and

$$
\forall u, \quad \lambda_{t}^{n} \Theta_{n}^{-1} \cdot u \text { } \sim e^{-\psi(u)}=a_{u}, \quad \text { as } n \rightarrow+\infty
$$

(where ${ }^{t} \Theta_{n}^{-1}$ stands for the transpose matrix of $\Theta_{n}^{-1}$ ) and

$$
\forall u \in[-b, b]^{d}, \quad\left|\lambda_{u}^{n}\right| \leq 2\left|e^{-\psi\left({ }^{t} \Theta_{n} \cdot u\right)}\right| .
$$

Note that, under Hypothesis 2.1 and if (14) holds true, then

$$
\forall u \in \mathbb{R}^{d}, \quad e^{-\psi(u)}=\lim _{n \rightarrow+\infty} \lambda_{t \Theta_{n}^{-1} \cdot u}^{n}=\lim _{n \rightarrow+\infty} \mathbb{E}_{\bar{\mu}}\left[P_{t^{\prime}}^{n} \cdot u=\lim _{n \rightarrow+\infty} \mathbb{E}_{\bar{\mu}}\left[e^{i u *\left(\Theta_{n}^{-1} S_{n}\right)}\right],\right.
$$

and so $\left(\Theta_{n}^{-1} S_{n}\right)_{n}$ converges in distribution to $Y$. If $Y$ has a stable distribution of index $\alpha \in$ $(0,2] \backslash\{1\}$, i.e.

$$
\psi(u)=\int_{\mathbb{S}^{1}}|u * s|^{\alpha}\left(1+\tan \frac{\pi}{\alpha} \operatorname{sign}(u * s)\right) d \Gamma(u),
$$

where $\Gamma$ is a Borel measure on the unit sphere $S^{1}=\left\{x \in \mathbb{R}^{d}: x * x=1\right\}$ and if

$$
\lambda_{u}=e^{-\psi(u) L\left(|u|^{-1}\right)}+o\left(|u|^{\alpha} L\left(|u|^{-1}\right)\right), \quad \text { as } u \rightarrow 0,
$$

with $L$ slowly varying at infinity, then Hypothesis 2.2 holds true with $\Theta_{n}:=\mathfrak{a}_{n} I d$ with $\mathfrak{a}_{n}:=$ $\inf \left\{x>0: n|x|^{-\alpha} L(x) \geq 1\right\}$.

But Hypothesis 2.2 allows also the study of situations with anisotropic scaling.

Before stating our first general result, let us introduce an additional notation. Under Hypothesis 2.1, for any function $u: \bar{M} \rightarrow \mathbb{C}$, we write $\|u\|_{\mathcal{B}_{0}^{\prime}}:=\sup _{h \in \mathcal{B}_{0}}\left|\mathbb{E}_{\bar{\mu}}[u . h]\right|$. 
Theorem 2.3. Assume Hypotheses 2.1 and 2.2. Let $f, g: M \rightarrow \mathbb{C}$ be such that

$$
\|f\|_{+}:=\sum_{\ell \in \mathbb{Z}^{d}}\|f(\cdot, \ell)\|<\infty \quad \text { and } \quad\|g\|_{+, \mathcal{B}_{0}^{\prime}}:=\sum_{\ell \in \mathbb{Z}^{d}}\|g(\cdot, \ell)\|_{\mathcal{B}_{0}^{\prime}}<\infty .
$$

Then

$$
\int_{M} f . g \circ T^{n} d \nu=\frac{\Phi(0)}{\operatorname{det} \Theta_{n}}\left(\int_{M} f d \nu \int_{M} g d \nu+o(1)\right) \text {, as } n \rightarrow+\infty \text {. }
$$

Proof. For every positive integer $n$ and every $\ell \in \mathbb{Z}^{d}$, combining (10) with Hypothesis 2.1, the following equalities hold in $\mathcal{L}\left(\mathcal{B}, \mathcal{B}_{0}\right)$ :

$$
\begin{aligned}
P^{n}\left(\mathbf{1}_{S_{n}=\ell}\right) & =\frac{1}{(2 \pi)^{d}} \int_{[-b, b]^{d}} e^{-i t * \ell} \lambda_{t}^{n} \Pi_{t}(\cdot) d t+O\left(\vartheta^{n}\right) \\
& =\frac{1}{(2 \pi)^{d} \operatorname{det} \Theta_{n}} \int_{{ }^{t} \Theta_{n}[-b, b]^{d}} e^{-i u *\left(\Theta_{n}^{-1} \ell\right)} \lambda_{t \Theta_{n}^{-1} u}^{n} \Pi_{t^{-1} u}(\cdot) d u+O\left(\vartheta^{n}\right) \\
& =\frac{1}{(2 \pi)^{d} \operatorname{det} \Theta_{n}} \int_{\mathbb{R}^{d}} e^{-i u *\left(\Theta_{n}^{-1} \ell\right)} e^{-\psi(u)} \Pi_{0}(\cdot) d u+\varepsilon_{n, \ell} \\
& =\frac{\Phi\left(\Theta_{n}^{-1} \ell\right)}{\operatorname{det} \Theta_{n}} \Pi_{0}+\varepsilon_{n, \ell},
\end{aligned}
$$

with $\sup _{\ell}\left\|\varepsilon_{n, \ell}\right\|_{\mathcal{L}\left(\mathcal{B}, \mathcal{B}_{0}\right)}=o\left(\operatorname{det} \Theta_{n}^{-1}\right)$ due to the dominated convergence theorem applied to

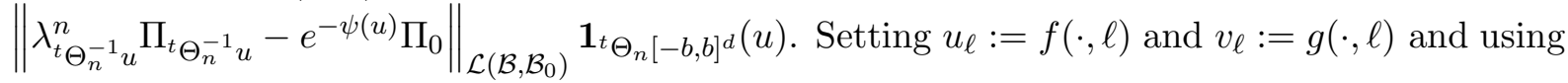
(9), we obtain

$$
\begin{aligned}
\int_{M} f . g \circ T^{n} d \nu & =\sum_{\ell, \ell^{\prime} \in \mathbb{Z}^{d}}\left(\frac{\Phi\left(\Theta_{n}^{-1}\left(\ell^{\prime}-\ell\right)\right)}{\operatorname{det} \Theta_{n}} \mathbb{E}_{\bar{\mu}}\left[u_{\ell}\right] \mathbb{E}_{\bar{\mu}}\left[v_{\ell^{\prime}}\right]+\mathbb{E}_{\bar{\mu}}\left[v_{\ell^{\prime}} \varepsilon_{n, \ell}\left(u_{\ell}\right)\right]\right) \\
& =\sum_{\ell, \ell^{\prime} \in \mathbb{Z}^{d}}\left(\frac{\Phi\left(\Theta_{n}^{-1}\left(\ell^{\prime}-\ell\right)\right)}{\operatorname{det} \Theta_{n}} \mathbb{E}_{\bar{\mu}}\left[u_{\ell}\right] \mathbb{E}_{\bar{\mu}}\left[v_{\ell^{\prime}}\right]\right)+O\left(\sum_{\ell, \ell^{\prime} \in \mathbb{Z}^{d}}\left\|v_{\ell^{\prime}}\right\|_{\mathcal{B}_{0}^{\prime}}\left\|\varepsilon_{n, \ell}\right\|_{\mathcal{L}\left(\mathcal{B}, \mathcal{B}_{0}\right)}\left\|u_{\ell}\right\|\right) \\
& =\sum_{\ell, \ell^{\prime} \in \mathbb{Z}^{d}} \frac{\Phi\left(\Theta_{n}^{-1}\left(\ell^{\prime}-\ell\right)\right)}{\operatorname{det} \Theta_{n}} \mathbb{E}_{\bar{\mu}}\left[u_{\ell}\right] \mathbb{E}_{\bar{\mu}}\left[v_{\ell^{\prime}}\right]+\tilde{\varepsilon}_{n}(f, g)
\end{aligned}
$$

with $\lim _{n \rightarrow+\infty} \sup _{f, g} \frac{\operatorname{det} \Theta_{n} \tilde{\varepsilon}_{n}(f, g)}{\|g\|_{+, \mathcal{B}_{0}^{\prime}}^{\prime}\|f\|_{+}}=0$. Now, due to the dominated convergence theorem and since $\Phi$ is continuous and bounded,

$$
\lim _{n \rightarrow+\infty} \sum_{\ell, \ell^{\prime} \in \mathbb{Z}^{d}} \Phi\left(\Theta_{n}^{-1}\left(\ell^{\prime}-\ell\right)\right) \mathbb{E}_{\bar{\mu}}\left[u_{\ell}\right] \mathbb{E}_{\bar{\mu}}\left[v_{\ell^{\prime}}\right]=\Phi(0) \sum_{\ell, \ell^{\prime} \in \mathbb{Z}^{2}} \mathbb{E}_{\bar{\mu}}\left[u_{\ell}\right] \mathbb{E}_{\bar{\mu}}\left[v_{\ell^{\prime}}\right]=\Phi(0) \int_{M} f d \nu \int_{M} g d \nu
$$

which ends the proof.

We will reinforce Hypothesis 2.2. Notations $\lambda_{0}^{(k)}, a_{0}^{(k)}, \Pi_{0}^{(k)}$ stand for the $k$-th derivatives of $\lambda, a$ and $\Pi$ at 0 .

Theorem 2.4. Assume Hypothesis $\left[2.1\right.$ with $\mathcal{B}_{0}=\mathcal{B}$. Let $K, M, P$ be three integers such that $K \geq d / 2,3 \leq P \leq M+1$ and

$$
-\left\lfloor\frac{M}{P}\right\rfloor+\frac{M}{2} \geq K
$$

Assume moreover that $\lambda$. is $C^{M}$-smooth and that there exists a positive symmetric matrix $\Sigma^{2}$ such that

$$
\lambda_{u}-1 \sim-\psi(u):=-\frac{1}{2} \Sigma^{2} * u^{\otimes 2}, \quad \text { as } u \rightarrow 0 .
$$


Assume that, for every $k<P, \lambda_{0}^{(k)}=a_{0}^{(k)}$ with $a_{t}=e^{-\psi(t)}$, for every $k<P$. Assume moreover that the functions $\Pi$ and $R$ are $C^{2 K}$-smooth. Let $f, g: M \rightarrow \mathbb{C}$ be such that

$$
\sum_{\ell \in \mathbb{Z}^{d}}\left(\|f(\cdot, \ell)\|+\|g(\cdot, \ell)\|_{\mathcal{B}^{\prime}}\right)<\infty
$$

Then

$$
\int_{M} f . g \circ T^{n} d \nu=\sum_{\ell, \ell^{\prime} \in \mathbb{Z}^{d}} \sum_{m=0}^{2 K} \frac{1}{m !} \sum_{j=0}^{M} \frac{i^{m+j}}{(j) !} \frac{\Phi^{(m+j)}\left(\frac{\ell^{\prime}-\ell}{\mathfrak{a}_{n}}\right)}{n^{\frac{d+m+j}{2}}} *\left(\mathbb{E}_{\bar{\mu}}\left[v_{\ell^{\prime}} \Pi_{0}^{(m)}\left(u_{\ell}\right)\right] \otimes\left(\lambda^{n} / a^{n}\right)_{0}^{(j)}\right)+o\left(n^{-K-\frac{d}{2}}\right) .
$$

If moreover $\sum_{\ell \in \mathbb{Z}^{d}}|\ell|^{2 K}\left(\|f(\cdot, \ell)\|+\|g(\cdot, \ell)\|_{\mathcal{B}^{\prime}}\right)<\infty$, then

$$
\begin{aligned}
\int_{M} f . g \circ T^{n} d \nu= & \sum_{m, j, r} \frac{i^{j+m}}{m ! r ! j !}\left(\frac{\Phi^{(j+m+r)}(0)}{n^{\frac{j+d+m+r}{2}}} *\left(\lambda^{n} / a^{n}\right)_{0}^{(j)}\right) \\
& * \sum_{\ell, \ell^{\prime} \in \mathbb{Z}^{d}}\left(\ell^{\prime}-\ell\right)^{\otimes r} \otimes \mathbb{E}_{\bar{\mu}}\left[v_{\ell^{\prime}} \Pi_{0}^{(m)}\left(u_{\ell}\right)\right]+o\left(n^{-K-\frac{d}{2}}\right),
\end{aligned}
$$

where the sum is taken over the $(m, j, r)$ with $m, j, r$ non negative integers such that $j+m+r \in 2 \mathbb{Z}$ and $\frac{r+m+j}{2}-\left\lfloor\frac{j}{P}\right\rfloor \leq K$.

Observe that

$$
\left(\lambda^{n} / a^{n}\right)_{0}^{(j)}=\sum_{k_{1} m_{1}+\ldots+k_{r} m_{r}=j} \frac{n !}{m_{1} ! \cdots m_{r} !\left(n-m_{1}-\ldots-m_{r}\right) !}\left((\lambda / a)_{0}^{\left(k_{1}\right)}\right)^{m_{1}} \cdots\left((\lambda / a)_{0}^{\left(k_{r}\right)}\right)^{m_{r}},
$$

where the sum is taken over $r \geq 1, m_{1}, \ldots, m_{r} \geq 1, k_{r}>\ldots>k_{1} \geq P$ (this implies that $\left.m_{1}+\ldots+m_{r} \leq j / P\right)$. Hence $\left(\lambda^{n} / a^{n}\right)_{0}^{(j)}$ is polynomial in $n$ with degree at most $\lfloor j / P\rfloor$.

Remark 2.5. Note that (17) holds true as soon as $M \geq 2 K P /(P-2)$ and $M$ in (20) can be replaced by $(2 K-m) P /(P-2)$.

Moreover (21) provides an expansion of the following form:

$$
\int_{M} f . g \circ T^{n} d \nu=\sum_{m=0}^{K} \frac{c_{m}(f, g)}{n^{\frac{d}{2}+m}}+o\left(n^{-K-\frac{d}{2}}\right) .
$$

Remark 2.6. If $\Pi$ is $C^{M}$-smooth, using the fact $\left(\lambda^{n} / a^{n}\right)_{0}^{(j)}=O\left(n^{\lfloor j / P\rfloor}\right)$, if $\sum_{\ell \in \mathbb{Z}^{d}}|\ell|^{M}(\|f(\cdot, \ell)\|+$ $\left.\|g(\cdot, \ell)\|_{\mathcal{B}^{\prime}}\right)<\infty$ the right hand side of (21) can be rewritten

$$
\frac{1}{n^{\frac{d}{2}}} \sum_{\ell, \ell^{\prime} \in \mathbb{Z}^{d}} \sum_{L=0}^{M} \frac{1}{n^{L / 2}} \frac{\Phi^{(L)}(0)}{L !} i^{L} \frac{\partial^{L}}{\partial t^{L}}\left(\mathbb{E}_{\bar{\mu}}\left[v_{\ell^{\prime}} \cdot e^{-i t *\left(\ell^{\prime}-\ell\right)} \cdot \lambda_{t}^{n} \Pi_{t} \cdot u_{\ell}\right] e^{\frac{n}{2} \Sigma^{2} * t^{\otimes 2}}\right)_{\mid t=0}+o\left(n^{-K-\frac{d}{2}}\right) .
$$

If moreover $\sup _{u \in[-b, b]^{d}}\left\|\left(R_{u}^{n}\right)^{(m)}\right\|_{(\mathcal{B}, \mathcal{B})}=O\left(\vartheta^{n}\right)$ for every $m=0, \ldots, M$, then it can also be rewritten

$$
\frac{1}{n^{\frac{d}{2}}} \sum_{\ell, \ell^{\prime} \in \mathbb{Z}^{d}} \sum_{L=0}^{M} \frac{\Phi^{(L)}(0)}{L !} i^{L} \frac{\partial^{L}}{\partial t^{L}}\left(\mathbb{E}_{\bar{\mu}}\left[u_{\ell} \cdot e^{i t * \frac{S_{n}-\left(\ell^{\prime}-\ell\right)}{\sqrt{n}}} \cdot v_{\ell^{\prime}} \circ \bar{T}^{n}\right] e^{\frac{1}{2} \Sigma^{2} * t^{\otimes 2}}\right)_{\mid t=0}+o\left(n^{-K-\frac{d}{2}}\right),
$$

where we used (13). 
Proof of Theorem 2.4. We assume, up to a change of $b$ that Hypothesis 2.2 holds true. Due to (10) and to (13), in $\mathcal{L}(\mathcal{B}, \mathcal{B})$, we have

$$
\begin{aligned}
P^{n}\left(\mathbf{1}_{S_{n}=\ell}\right) & =\frac{1}{(2 \pi)^{d}} \int_{[-\pi, \pi]^{d}} e^{-i t * \ell} P_{t}^{n}(\cdot) d t \\
& =\frac{1}{(2 \pi)^{d}} \int_{[-b, b]^{d}} e^{-i t * \ell} \lambda_{t}^{n} \Pi_{t}(\cdot) d t+O\left(\vartheta^{n}\right) \\
& =\frac{1}{(2 \pi)^{d} n^{\frac{d}{2}}} \int_{[-b \sqrt{n}, b \sqrt{n}]^{d}} e^{-i t * \frac{\ell}{\sqrt{n}}} \lambda_{t / \sqrt{n}}^{n} \Pi_{t / \sqrt{n}(\cdot) d t+O\left(\vartheta^{n}\right)} \\
& =\frac{1}{(2 \pi)^{d} n^{\frac{d}{2}}} \int_{[-b \sqrt{n}, b \sqrt{n}]^{d}} e^{-i t * \frac{\ell}{\sqrt{n}}} \lambda_{t / \sqrt{n}}^{n} \sum_{m=0}^{2 K} \frac{1}{m !} \Pi_{0}^{(m)}(\cdot) * \frac{t^{\otimes m}}{n^{\frac{m}{2}}} d t+o\left(n^{-K-\frac{d}{2}}\right),
\end{aligned}
$$

due to the dominated convergence theorem since there exists $x_{t / \sqrt{n}} \in(0, t / \sqrt{n})$ such that $\Pi_{t / \sqrt{n}}(\cdot)=\sum_{m=0}^{2 K-1} \frac{1}{m !} \Pi_{0}^{(m)}(\cdot) * \frac{t^{\otimes m}}{n^{\frac{m}{2}}}+\frac{1}{(2 K) !} \Pi_{0}^{(2 K)}\left(x_{t / \sqrt{n}}\right) * \frac{t^{\otimes 2 K}}{n^{K}}$. Recall that $\left(\lambda^{n} / a^{n}\right)_{0}^{(j)}=O\left(n^{\lfloor j / P\rfloor}\right)$, so

$$
\left|\lambda_{t / \sqrt{n}}^{n}-a_{t} \sum_{j=0}^{M} \frac{1}{j !}\left(\lambda^{n} / a^{n}\right)_{0}^{(j)} * \frac{t^{\otimes j}}{n^{\frac{j}{2}}}\right| \leq n^{\left\lfloor\frac{M}{P}\right\rfloor} a_{t} \frac{|t|^{M}}{n^{\frac{M}{2}}} \eta(t / \sqrt{n}),
$$

with $\lim _{t \rightarrow 0} \eta(t)=0$ and $\sup _{[-b, b]^{d}}|\eta|<\infty$. Due to (17), we obtain

$$
\begin{aligned}
P^{n}\left(\mathbf{1}_{S_{n}=\ell}\right)= & \frac{1}{(2 \pi)^{d} n^{\frac{d}{2}}} \int_{[-b \sqrt{n}, b \sqrt{n}]^{d}} e^{-i t * \frac{\ell}{\sqrt{n}}} e^{-\frac{1}{2} \Sigma^{2} * t^{\otimes 2}} \sum_{m=0}^{2 K} \frac{1}{m !} \Pi_{0}^{(m)}(\cdot) * \frac{t^{\otimes m}}{n^{\frac{m}{2}}} \\
& \left(1+\sum_{j=P}^{M} \frac{1}{j !}\left(\lambda^{n} / a^{n}\right)_{0}^{(j)} * \frac{t^{\otimes j}}{n^{\frac{j}{2}}}\right) d t+o\left(n^{-K-\frac{d}{2}}\right) \\
= & \sum_{m=0}^{2 K} \sum_{j=0}^{M} \frac{i^{m+j}}{n^{\frac{m+j+d}{2}} m ! j !} \Phi^{(m+j)}\left(\frac{\ell}{\sqrt{n}}\right) *\left(\Pi_{0}^{(m)}(\cdot) \otimes\left(\lambda^{n} / a^{n}\right)_{0}^{(j)}\right)+o\left(n^{-K-\frac{d}{2}}\right) .
\end{aligned}
$$

This combined with (9) and (19) gives (20).

We assume from now on that $\sum_{\ell \in \mathbb{Z}^{d}}|\ell|^{2 K}\left(\|f(\cdot, \ell)\|+\|g(\cdot, \ell)\|_{\mathcal{B}^{\prime}}\right)$. Recall that $\left(\lambda^{n} / a^{n}\right)_{0}^{(j)}$ is polynomial in $n$ of degree at most $\lfloor j / P\rfloor$. Hence, due to the dominated convergence theorem, we can replace $\Phi^{(m+j)}\left(\frac{\ell^{\prime}-\ell}{\sqrt{n}}\right)$ in (20) by

$$
\sum_{r=0}^{2 K-m-j+2\left\lfloor\frac{j}{P}\right\rfloor} \frac{1}{r ! n^{\frac{r}{2}}} \Phi^{(m+j+r)}(0) *\left(\ell^{\prime}-\ell\right)^{\otimes r} .
$$

Hence we have proved (21).

Now, we come back to the case of $\mathbb{Z}^{2}$-periodic Sinai billiards, with the notations of Section 1 .

\section{YOUNG TOWERS FOR BILLIARDS}

Recall that, in [30], Young constructed two dynamical systems $(\tilde{M}, \tilde{T}, \tilde{\mu})$ and $(\hat{M}, \hat{T}, \hat{\mu})$ and two measurable functions $\tilde{\pi}: \tilde{M} \rightarrow \bar{M}$ and $\hat{\pi}: \tilde{M} \rightarrow \hat{M}$ such that

$$
\tilde{\pi} \circ \tilde{T}=\bar{T} \circ \tilde{\pi}, \tilde{\pi}_{*} \tilde{\mu}=\bar{\mu}, \hat{\pi} \circ \tilde{T}=\hat{T} \circ \hat{\pi}, \hat{\pi}_{*} \tilde{\mu}=\hat{\mu}
$$

and such that, for every measurable $f: \bar{M} \rightarrow \mathbb{C}$ constant on every stable manifold, there exists $\hat{f}: \hat{M} \rightarrow \mathbb{C}$ such that $\hat{f} \circ \hat{\pi}=f \circ \tilde{\pi}$. We consider the partition $\hat{\mathcal{D}}$ on $\hat{M}$ constructed by Young in 
[30] together with the separation time given, for every $x, y$, by

$$
s_{0}(x, y):=\min \left\{n \geq-1: \hat{\mathcal{D}}\left(\hat{T}^{n+1} x\right) \neq \hat{\mathcal{D}}\left(\hat{T}^{n+1} y\right)\right\} .
$$

It will be worth noting that, for any $x, y$, the sets $\tilde{\pi} \hat{\pi}^{-1}\{x\}$ and $\tilde{\pi} \hat{\pi}^{-1}\{y\}$ are contained in the same connected component of $\bar{M} \backslash \bigcup_{k=0}^{s_{0}(x, y)} \bar{T}^{-k} \mathcal{S}_{0}$.

Let $p>1$ and set $q$ such that $\frac{1}{p}+\frac{1}{q}=1$. Let $\varepsilon>0$ and $\beta \in(0,1)$ be suitably chosen and let us define

$$
\|\hat{f}\|=\sup _{\ell}\left\|\hat{f}_{\mid \hat{\Delta}_{\ell}}\right\|_{\infty} e^{-\ell \varepsilon}+\sup _{\hat{A} \in \hat{\mathcal{D}}} \operatorname{esssup}_{x, y \in \hat{A}} \frac{|\hat{f}(x)-\hat{f}(y)|}{\beta^{s_{0}(x, y)}} e^{-\ell \varepsilon} .
$$

Let $\mathcal{B}:=\left\{\hat{f} \in L_{\mathbb{C}}^{q}(\hat{M}, \hat{\mu}):\|\hat{f}\|<\infty\right\}$. Young proved that the Banach space $(\mathcal{B},\|\cdot\|)$ satisfies $\|\cdot\|_{q} \leq\|\cdot\|$, that the transfer opertor $\hat{P}$ on $\mathcal{B}\left(\hat{P}\right.$ being defined on $L^{q}$ as the adjoint of the composition by $\hat{T}$ on $L^{p}$ ) is quasicompact on $\mathcal{B}$. We assume without any loss of generality (up to an adaptation of the construction of the tower) that the dominating eigenvalue of $\hat{P}$ on $\mathcal{B}$ is 1 and is simple.

Since $\kappa: \bar{M} \rightarrow \mathbb{Z}^{2}$ is constant on the stable manifolds, there exists $\hat{\kappa}: \hat{M} \rightarrow \mathbb{Z}^{2}$ such that $\hat{\kappa} \circ \hat{\pi}=\kappa \circ \tilde{\pi}$. We set $\hat{S}_{n}:=\sum_{k=0}^{n-1} \hat{\kappa} \circ \hat{T}^{k}$. For any $u \in \mathbb{R}^{2}$ and $\hat{f} \in \mathcal{B}$, we set $\hat{P}_{u}(\hat{f}):=\hat{P}\left(e^{i u * \hat{\kappa}} \hat{f}\right)$.

Proposition 3.1. $t \mapsto \lambda_{t}$ is an even function.

Proof. Let $\Psi: \bar{M} \rightarrow \bar{M}$ be the map which sends $(q, \vec{v}) \in \bar{M}$ to $\left(q, \vec{v}^{\prime}\right) \in \bar{M}$ such that $\left(\widehat{\left.\vec{n}(q), \vec{v}^{\prime}\right)=}\right.$ $-(\widehat{\vec{n}(q), \vec{v}})$. Then $\kappa \circ \bar{T}^{k} \circ \Psi=-\kappa \circ \bar{T}^{-k-1}$. Hence, $S_{n}$ as the same distribution (with respect to $\bar{\mu})$ as $-S_{n}$ and so

$$
\forall t \in[-b, b]^{2}, \quad \mathbb{E}_{\mu}\left[e^{-i t * S_{n}}\right]=\mathbb{E}_{\mu}\left[e^{i t * S_{n}}\right] \sim \lambda_{t}^{n} \mathbb{E}_{\hat{\mu}}\left[\Pi_{t} \mathbf{1}\right] \sim \lambda_{-t}^{n} \mathbb{E}_{\hat{\mu}}\left[\Pi_{-t} \mathbf{1}\right]
$$

as $n$ goes to infinity, and so $\lambda$ is even.

Let $\mathcal{Z}_{k}^{m}$ be the partition of $\bar{M} \backslash \bigcup_{j=k}^{m} \bar{T}^{-j}\left(\mathcal{S}_{0}\right)$ into its connected components. We also write $\mathcal{Z}_{k}^{\infty}:=\bigvee_{j \geq k} \mathcal{Z}_{k}^{j}$.

Proposition 3.2. Let $k$ be a nonnegative integer and let $u, v: \bar{M} \rightarrow \mathbb{C}$ be respectively $\mathcal{Z}_{-k^{-}}^{k}$ measurable and $Z_{-k}^{\infty}$-measurable functions.

Then there exists $\hat{u}, \hat{v}: \hat{M} \rightarrow \mathbb{C}$ such that $u \circ \bar{T}^{k} \circ \tilde{\pi}=\hat{u} \circ \hat{\pi}$ and $v \circ \bar{T}^{k} \circ \tilde{\pi}=\hat{v} \circ \hat{\pi}$.

Moreover, $\hat{u} \in \mathcal{B}$ and for every $t \in \mathbb{R}, \hat{P}_{t}^{2 k}\left(e^{-i t * \hat{S}_{k}} \hat{u}\right)=\hat{P}^{2 k}\left(e^{i t * \hat{S}_{k} \circ \hat{T}^{k}} \hat{u}\right)$ and

$$
\left\|\hat{P}^{2 k}\left(e^{i t * \hat{S}_{k} \circ \hat{T}^{k}} \hat{u}\right)\right\| \leq\left(1+2 \beta^{-1}\right)\|u\|_{\infty},
$$

and

$$
\forall n>k, \quad \mathbb{E}_{\bar{\mu}}\left[u \cdot e^{i t * S_{n}} \cdot v \circ \bar{T}^{n}\right]=\mathbb{E}_{\hat{\mu}}\left[\hat{v} \cdot e^{i t * \hat{S}_{k}} \hat{P}_{t}^{n}\left(e^{-i t * \hat{S}_{k}} \hat{u}\right)\right] .
$$

Proof. Using several times $\hat{P}^{m}\left(f . g \circ T^{m}\right)=g \cdot \hat{P}^{m} f$ and $\hat{P}_{t}^{m}=\hat{P}^{m}\left(e^{i t \hat{S}_{m}} \cdot\right)$, we obtain

$$
\begin{aligned}
\mathbb{E}_{\bar{\mu}}\left[u . e^{i t * S_{n}} \cdot v \circ \bar{T}^{n}\right] & =\mathbb{E}_{\bar{\mu}}\left[u \circ \bar{T}^{k} \cdot e^{i t * S_{n}} \circ \bar{T}^{k} \cdot v \circ \bar{T}^{n+k}\right] \\
& =\mathbb{E}_{\hat{\mu}}\left[\hat{u} \cdot e^{i t * \hat{S}_{n}} \circ \hat{T}^{k} \cdot \hat{v} \circ \hat{T}^{n}\right] \\
& =\mathbb{E}_{\hat{\mu}}\left[\hat{P}^{n+k}\left(\hat{u} \cdot e^{i t *\left(\hat{S}_{n-k} \circ \hat{T}^{k}+\hat{S}_{k} \circ \hat{T}^{n}\right)} \cdot \hat{v} \circ \hat{T}^{n}\right)\right] \\
& =\mathbb{E}_{\hat{\mu}}\left[\hat{P}^{k}\left(e^{i t * \hat{S}_{k}} \hat{v} \cdot \hat{P}^{n}\left(e^{i t *\left(\hat{S}_{n-k} \circ \hat{T}^{k}\right)} \cdot \hat{u}\right)\right)\right] \\
& =\mathbb{E}_{\hat{\mu}}\left[\hat{P}^{k}\left(e^{i t * \hat{S}_{k}} \hat{v} \cdot \hat{P}_{t}^{n}\left(e^{-i t * \hat{S}_{k}} \hat{u}\right)\right)\right],
\end{aligned}
$$

since $\hat{S}_{n-k} \circ \hat{T}^{k}=\hat{S}_{n}-\hat{S}_{k}$. Hence, we have proved (23) (since $\hat{P}$ preserves $\hat{\mu}$ ). 


\section{Proofs of our main Results in the Finite horizon CASE}

We assume throughout this section that the billiard has finite horizon.

The Nagaev-Guivarc'h method [16, 17, 11] has been applied in this context by Szász and Varjú [27] (see also [19]) to prove Hypotheses 2.1 and 2.2 hold for $\mathcal{B}_{0}=\mathcal{B}$ the Young Banach space. More precisely, we have the following.

Proposition 4.1 ([27, 19]). There exist a real $b \in(0, \pi)$ and three $C^{\infty}$ functions $t \mapsto \lambda_{t}, t \mapsto \Pi_{t}$ and $t \mapsto N_{t}$ defined on $[-b, b]^{2}$ and with values in $\mathbb{C}, \mathcal{L}(\mathcal{B}, \mathcal{B})$ and $\mathcal{L}(\mathcal{B}, \mathcal{B})$ respectively such that

(i) for every $t \in[-b, b]^{2}, \hat{P}_{t}^{n}=\lambda_{t}^{n} \Pi_{t}+N_{t}^{n}$ and $\Pi_{0}=\mathbb{E}_{\hat{\mu}}[\cdot], \Pi_{t} \hat{P}_{t}=\hat{P}_{t} \Pi_{t}=\lambda_{t} \Pi_{t}, \Pi_{t}^{2}=\Pi_{t}$;

(ii) there exists $\vartheta \in(0,1)$ such that, for every positive integer $m$,

$$
\sup _{t \in[-b, b]^{2}}\left\|\left(N^{n}\right)_{t}^{(m)}\right\|_{\mathcal{L}(\mathcal{B}, \mathcal{B})}=O\left(\vartheta^{n}\right) \quad \text { and } \sup _{t \in[-\pi, \pi]^{2} \backslash[-b, b]^{2}}\left\|\hat{P}_{t}^{n}\right\|_{\mathcal{L}(\mathcal{B}, \mathcal{B})}=O\left(\vartheta^{n}\right)
$$

(iii) we have $\lambda_{t}=1-\frac{1}{2} \Sigma^{2} * t^{\otimes 2}=O\left(|t|^{3}\right)$;

(iv) there exists $\sigma>0$ such that, for any $t \in[-b, b]^{2},\left|\lambda_{t}\right| \leq e^{-\sigma|t|^{2}}$ and $e^{-\frac{1}{2} \Sigma^{2} * t^{\otimes 2}} \leq e^{-\sigma|t|^{2}}$.

Our first step consists in stating a high order expansion of the following quantity

$$
\mathbb{E}_{\bar{\mu}}\left[u .1_{S_{n}=\ell} \cdot v \circ \bar{T}^{n}\right]
$$

for $u$ and $v$ dynamically Lispchitz on $\bar{M}$. Let us recall that, due to (8), this result corresponds to a mixing result for observables supported on a single cell. We start by studying this quantity for some locally constant observables. This result is a refinement of [22, prop. 4.1] (see also [21, prop 3.1]. Let $\Phi$ be the density function of $B$, which is given by $\Phi(x)=\frac{e^{-\frac{\left(\Sigma^{2}\right)^{-1} * x}{2} 2_{2}}}{2 \pi \sqrt{\operatorname{det} \Sigma^{2}}}$.

4.1. A first local limit theorem. We set $a_{t}:=e^{-\frac{1}{2} \Sigma^{2} * t^{\otimes 2}}$. Note that the uneven derivatives of $\lambda / a$ at 0 are null as well as its three first derivatives.

Proposition 4.2. Let $K$ be a positive integer and a real number $p>1$. There exists $c>0$ such that, for any $k \geq 1$, if $u, v: \bar{M} \rightarrow \mathbb{C}$ are respectively $\mathcal{Z}_{-k}^{k}$-measurable and $\mathcal{Z}_{-k}^{\infty}$-measurable, then for any $n>3 k$ and $\ell \in \mathbb{Z}^{2}$

$$
\begin{aligned}
& \left|\mathbb{E}_{\bar{\mu}}\left[u \mathbf{1}_{\left\{S_{n}=\ell\right\}} \cdot v \circ \bar{T}^{n}\right]-\sum_{m=0}^{2 K-2} \frac{1}{m !} \sum_{j=0}^{2 K-2-m} \frac{i^{m+2 j}}{(2 j) !} \frac{\Phi^{(m+2 j)}\left(\frac{\ell}{\sqrt{n}}\right)}{n^{j+1+\frac{m}{2}}} *\left(A_{m}(u, v) \otimes\left(\lambda^{n} / a^{n}\right)_{0}^{(2 j)}\right)\right| \\
& \leq \frac{c k^{2 K-1}\|v\|_{p}\|u\|_{\infty}}{n^{K+\frac{1}{4}}},
\end{aligned}
$$

with, for every $m \in\{0, \ldots, 4 K-4\}$,

$$
\begin{gathered}
\left|A_{m}(u, v)-\frac{\partial^{m}}{\partial t^{m}}\left(\frac{\mathbb{E}_{\bar{\mu}}\left[u \cdot e^{i t * S_{n}} \cdot v \circ \bar{T}^{n}\right]}{\lambda_{t}^{n}}\right)_{\mid t=0}\right| \leq c n^{m} \vartheta^{n-2 k}\|v\|_{p}\|u\|_{\infty}, \\
\left|A_{m}(u, v)\right| \leq c k^{m}\|v\|_{p}\|u\|_{\infty} \quad \text { and } \quad\left(\lambda^{n} / a^{n}\right)_{0}^{(m)}=O\left(n^{m / 4}\right) .
\end{gathered}
$$

In particular, for $K=2$, we obtain

$$
\begin{aligned}
& \mid \mathbb{E}_{\bar{\mu}}\left[u \mathbf{1}_{\left\{S_{n}=\ell\right\}} \cdot v \circ \bar{T}^{n}\right]-\frac{\Phi\left(\frac{\ell}{\sqrt{n}}\right)}{n} A_{0}(u, v)-\frac{i}{n^{\frac{3}{2}}} \Phi^{\prime}\left(\frac{\ell}{\sqrt{n}}\right) * A_{1}(u, v) \\
& +\frac{1}{n^{2}} \Phi "\left(\frac{\ell}{\sqrt{n}}\right) * A_{2}(u, v)-\frac{A_{0}(u, v)}{n^{2}} \cdot \Phi^{(4)}\left(\frac{\ell}{\sqrt{n}}\right) *\left(\lambda^{n} / a^{n}\right)_{0}^{(4)} \mid \\
& \leq \frac{c k^{3}\|v\|_{p}\|u\|_{\infty}}{n^{\frac{9}{4}}} .
\end{aligned}
$$


Remark 4.3. Due to (25) and (26), (24) can be rewritten as follows:

$$
\begin{aligned}
& \left|\mathbb{E}_{\bar{\mu}}\left[u \mathbf{1}_{\left\{S_{n}=\ell\right\}} \cdot v \circ \bar{T}^{n}\right]-\sum_{m=0}^{4 K-4} \frac{i^{m}}{m !} \frac{\Phi^{(m)}\left(\frac{\ell}{\sqrt{n}}\right)}{n^{1+\frac{m}{2}}} *\left(e^{\frac{n}{2} \Sigma^{2} * t^{\otimes 2}} \mathbb{E}_{\bar{\mu}}\left[u . e^{i t * S_{n}} \cdot v \circ \bar{T}^{n}\right]\right)_{\mid t=0}^{(m)}\right| \\
& \leq \frac{c k^{4 K-4}\|v\|_{p}\|u\|_{\infty}}{n^{K+\frac{1}{4}}} .
\end{aligned}
$$

Proof of Proposition 4.2. Since $u \circ \bar{T}^{k}$ is $\mathcal{Z}_{0}^{2 k}$-measurable and $v \circ \bar{T}^{k}$ is $\mathcal{Z}_{0}^{\infty}$-measurable, there exist $\hat{u}, \hat{v}: \hat{M} \rightarrow \mathbb{C}$ such that $\hat{u} \circ \hat{\pi}=u \circ \bar{T}^{k} \circ \tilde{\pi}$ and $\hat{v} \circ \hat{\pi}=v \circ \bar{T}^{k} \circ \tilde{\pi}$, with $\hat{u} \in \mathcal{B}$. As in the proof of [22, Prop. 4.1], we set

$$
C_{n}(u, v, \ell):=\mathbb{E}_{\bar{\mu}}\left[u .1_{\left\{S_{n}=\ell\right\}} \cdot v \circ \bar{T}^{n}\right] .
$$

Due to (23), we obtain

$$
\begin{aligned}
C_{n}(u, v, \ell) & =\frac{1}{(2 \pi)^{2}} \int_{[-\pi, \pi]^{2}} e^{-i t * \ell} \mathbb{E}_{\bar{\mu}}\left[u \cdot e^{i t * S_{n}} \cdot v \circ \bar{T}^{n}\right] d t \\
& =\frac{1}{(2 \pi)^{2}} \int_{[-\pi, \pi]^{2}} e^{-i t * \ell} \mathbb{E}_{\hat{\mu}}\left[e^{i t * \hat{S}_{k}} \hat{v} \cdot \hat{P}_{t}^{n}\left(e^{-i t * \hat{S}_{k}} \hat{u}\right)\right] d t .
\end{aligned}
$$

Let $\Xi_{k, t}:=e^{i t * \hat{S}_{k}} \prod_{t}\left(e^{-i t * \hat{S}_{k}}.\right)$. We will write $\Xi_{k, 0}^{(m)}$ for $\frac{\partial^{m}}{\partial t^{m}}\left(\Xi_{k, t}\right)_{\mid t=0}$. Due to items (i) and (ii) of Proposition 4.1 and due to (22), it comes

$$
\begin{aligned}
C_{n}(u, v, \ell) & =\frac{1}{(2 \pi)^{2}} \int_{[-b, b]^{2}} e^{-i t * \ell} \lambda_{t}^{n-2 k} \mathbb{E}_{\hat{\mu}}\left[e^{i t * \hat{S}_{k}} \hat{v} \cdot \Pi_{t} \hat{P}_{t}^{2 k}\left(e^{-i t * \hat{S}_{k}} \hat{u}\right)\right] d t+O\left(\vartheta^{n-2 k}\|u\|_{\infty} \cdot\|v\|_{p}\right) \\
& =\frac{1}{(2 \pi)^{2}} \int_{[-b, b]^{2}} e^{-i t * \ell} \lambda_{t}^{n} \mathbb{E}_{\hat{\mu}}\left[\hat{v} \cdot \Xi_{k, t} \hat{u}\right] d t+O\left(\vartheta^{n-2 k}\|u\|_{\infty} \cdot\|v\|_{p}\right)
\end{aligned}
$$

since $\Pi_{t} \hat{P}_{t}=\lambda_{t} \Pi_{t}$ and $\Pi_{t}^{2}=\Pi_{t}$ so that

$$
\Xi_{k, t}=\lambda_{t}^{-2 k} e^{i t * \hat{S}_{k}} \Pi_{t} \hat{P}_{t}^{2 k}\left(e^{-i t * \hat{S}_{k}} \cdot\right) .
$$

Observe that

$$
\frac{1}{(2 \pi)^{2}} \int_{[-b, b]^{2}}|t|^{j}\left|\lambda_{t}\right|^{n} d t \leq \frac{1}{(2 \pi)^{2} n^{\frac{j+2}{2}}} \int_{[-b \sqrt{n}, b \sqrt{n}]^{2}}|t|^{j} e^{-\sigma|t|^{2}} d t
$$

and so

$$
C_{n}(u, v, \ell)=\frac{1}{(2 \pi)^{2}} \int_{[-b, b]^{2}} e^{-i t * \ell} \lambda_{t}^{n} \sum_{m=0}^{2 K-2} \frac{1}{m !} A_{m}(u, v) * t^{\otimes m} d t+O\left(\frac{k^{2 K-1}\|v\|_{p}\|u\|_{\infty}}{n^{K+\frac{1}{2}}}\right),
$$

with $A_{m}(u, v):=\mathbb{E}_{\hat{\mu}}\left[\hat{v} \cdot \Xi_{k, 0}^{(m)} \hat{u}\right]$. Indeed $\Xi_{k, s}^{(2 K-1)} \hat{u}$ is a linear combination of terms of the form

$$
e^{i s * \hat{S}_{k}} \cdot\left(i \hat{S}_{k}\right)^{\otimes a} \otimes \Pi_{s}^{(b)} \hat{P}^{2 k}\left(\otimes\left(i \hat{S}_{k} \circ \hat{T}^{k}\right)^{\otimes c} e^{i s * \hat{S}_{k} \circ \hat{T}^{k}} \hat{u}\right) \otimes\left(\lambda^{-2 k}\right)_{s}^{(d)}
$$

over nonnegative integers $a, b, c, d$ such that $a+b+c+d=2 K-1$, and these terms are in $O\left(k^{2 K-1}\|u\|_{\infty}\right)$ in $\mathcal{B}$, uniformly in $k$. Moreover, due to (29), to (23) and to Item (i) of Proposition 4.1, we obtain

$$
\begin{aligned}
\forall t \in[-b, b]^{2}, \quad \mathbb{E}_{\hat{\mu}}\left[\hat{v} \cdot \Xi_{k, t} \hat{u}\right] & =\frac{\lambda_{t}^{n-2 k} \mathbb{E}_{\hat{\nu}}\left[\hat{v} \cdot e^{i t * \hat{S}_{k}} \Pi_{t} \hat{P}_{t}^{2 k}\left(e^{-i t * \hat{S}_{k}} \hat{u}\right)\right]}{\lambda_{t}^{n}} \\
& =\frac{\mathbb{E}_{\bar{\mu}}\left[u \cdot e^{i t * S_{n}} \cdot v \circ \bar{T}^{n}\right]-\mathbb{E}_{\hat{\mu}}\left[e^{i t * \hat{S}_{k}} \hat{v} \cdot N_{t}^{n-2 k} \hat{P}_{t}^{2 k}\left(e^{-i t * \hat{S}_{k}} \hat{u}\right)\right]}{\lambda_{t}^{n}},
\end{aligned}
$$


so that

$$
\mathbb{E}_{\hat{\mu}}\left[\hat{v} \cdot \Xi_{k, 0}^{(m)} \cdot \hat{u}\right]=\left(\frac{\mathbb{E}_{\bar{\mu}}\left[u \cdot e^{i t * S_{n}} \cdot v \circ \bar{T}^{n}\right]}{\lambda_{t}^{n}}\right)_{\mid t=0}^{(m)}+O\left(n^{m} \vartheta^{n-2 k}\|v\|_{p}\|u\|_{\infty}\right) .
$$

Recall that $a_{t}=e^{-\frac{1}{2} \Sigma^{2} * t^{\otimes 2}}$. Since the three first derivatives of $\lambda$ and $a$ coincide, we have $\left(\lambda^{n} / a^{n}\right)_{0}^{(j)}=O\left(n^{j / 4}\right)$ and

$$
\left|\lambda_{t}^{n}-a_{t}^{n} \sum_{j=0}^{4 K-4-2 m} \frac{1}{j !}\left(\lambda^{n} / a^{n}\right)_{0}^{(j)} * t^{\otimes j}\right| \leq c_{K} n^{\frac{4 K-3-2 m}{4}} a_{t}^{n}|t|^{4 K-3-2 m} .
$$

Due to the analogue of (301) with $\lambda_{t}$ replaced by $a_{t}$, we obtain

$$
\begin{aligned}
C_{n}(u, v, \ell)= & \frac{1}{(2 \pi)^{2}} \int_{[-b, b]^{2}} e^{-i t * \ell} e^{-\frac{n}{2} \Sigma^{2} * t^{\otimes 2}} \sum_{m=0}^{2 K-2} \frac{1}{m !} A_{m}(u, v) * t^{\otimes m} \\
& \left(1+\sum_{j=4}^{4 K-4-2 m} \frac{1}{j !}\left(\lambda^{n} / a^{n}\right)_{0}^{(j)} * t^{\otimes j}\right) d t+O\left(\frac{k^{2 K-1}\|v\|_{p}\|u\|_{\infty}}{n^{K+\frac{1}{4}}}\right) .
\end{aligned}
$$

Note that

$$
\begin{aligned}
& \frac{1}{(2 \pi)^{2}} \int_{[-b, b]^{2}} e^{-i t * \ell} e^{-\frac{n}{2} \Sigma^{2} * t^{\otimes 2}} t^{\otimes m} d t \\
= & \frac{1}{(2 \pi)^{2} n^{\frac{m}{2}+1}} \int_{[-b \sqrt{n}, b \sqrt{n}]^{2}} e^{-i t * \frac{\ell}{\sqrt{n}}} e^{-\frac{1}{2} \Sigma^{2} * t^{\otimes 2}} t^{\otimes m} d t \\
= & \frac{i^{m}}{n^{\frac{m}{2}+1}} \Phi^{(m)}\left(\frac{\ell}{\sqrt{n}}\right)+o\left(n^{-K-\frac{1}{4}}\right) .
\end{aligned}
$$

Hence we have proved that

$$
\begin{aligned}
& \mid \mathbb{E}_{\bar{\mu}}\left[u \mathbf{1}_{\left\{S_{n}=\ell\right\}} \cdot v \circ \bar{T}^{n}\right]-\sum_{m=0}^{2 K-2} \frac{i^{m}}{m !} \frac{\Phi^{(m)}\left(\frac{\ell}{\sqrt{n}}\right)}{n^{1+\frac{m}{2}}} * A_{m}(u, v) \\
& -\sum_{m=0}^{2 K-2} \sum_{j=4}^{4 K-4-2 m} \frac{i^{m+j}}{m ! j ! n^{1+\frac{m+j}{2}}} \Phi^{(m+j)}\left(\frac{\ell}{\sqrt{n}}\right) *\left(A_{m}(u, v) \otimes\left(\lambda^{n} / a^{n}\right)_{0}^{(j)}\right) \mid \\
& \leq \frac{c k^{2 K-1}\|v\|_{p}\|u\|_{\infty}}{n^{K+\frac{1}{4}}}
\end{aligned}
$$

and so (24) using (32) and the fact that the uneven derivatives of $(\lambda / a)$ at 0 are null.

\subsection{Generalization.}

Proposition 4.4. Let $K$ be a positive integer. Let $\xi_{0} \in(\max (\xi, \vartheta), 1)$. There exists $\mathfrak{c}_{0}>0$ such that, for every $u, v: \bar{M} \rightarrow \mathbb{C}$ dynamically Lipschitz continuous functions, with respect to $d_{\xi}$ with $\xi \in(0,1)$ and for every $\ell \in \mathbb{Z}^{2}$

$$
\begin{aligned}
& \left|\mathbb{E}_{\bar{\mu}}\left[u \mathbf{1}_{\left\{S_{n}=\ell\right\}} \cdot v \circ \bar{T}^{n}\right]-\sum_{m=0}^{2 K-2} \frac{1}{m !} \sum_{j=0}^{2 K-2-m} \frac{i^{m+2 j}}{(2 j) !} \frac{\Phi^{(m+2 j)}\left(\frac{\ell}{\sqrt{n}}\right)}{n^{j+1+\frac{m}{2}}} *\left(A_{m}(u, v) \otimes\left(\lambda^{n} / a^{n}\right)_{0}^{(2 j)}\right)\right| \\
& \leq \mathfrak{c}_{0} \frac{(\log n)^{4 K-2}}{n^{K+\frac{1}{4}}}\|v\|_{(\xi)}\|u\|_{(\xi)},
\end{aligned}
$$


with $A_{m}(u, v)$ such that

$$
\left|A_{m}(u, v)-\left(\mathbb{E}_{\bar{\mu}}\left[u . e^{i t * S_{n}} \cdot v \circ \bar{T}^{n}\right] / \lambda_{t}^{n}\right)_{\mid t=0}^{(m)}\right| \leq \mathfrak{c}_{0}\|u\|_{(\xi)}\|u\|_{(\xi)} \xi_{0}^{(\log n)^{2}}
$$

and $\left|A_{m}(u, v)\right| \leq \mathfrak{c}_{0}\|u\|_{(\xi)}\|v\|_{(\xi)}$.

Proof. For every positive integer $k$, we define

$$
u_{k}:=\mathbb{E}_{\bar{\mu}}\left[u \mid \mathcal{Z}_{-k}^{k}\right] \quad \text { and } \quad v_{k}:=\mathbb{E}\left[v \mid \mathcal{Z}_{-k}^{k}\right]
$$

Note that

$$
\left\|u-u_{k}\right\|_{\infty} \leq L_{\xi}(u) \xi^{k}, \quad\left\|v-v_{k}\right\|_{\infty} \leq L_{\xi}(v) \xi^{k}
$$

and

$$
\left|\mathbb{E}_{\bar{\mu}}\left[u \mathbf{1}_{\left\{S_{n}=\ell\right\}} \cdot v \circ \bar{T}^{n}\right]-\mathbb{E}_{\bar{\mu}}\left[u_{k} \mathbf{1}_{\left\{S_{n}=\ell\right\}} \cdot v_{k} \circ \bar{T}^{n}\right]\right| \leq\|u\|_{(\xi)}\|v\|_{(\xi)} \xi^{k} .
$$

Now we take $k=k_{n}=\left\lceil(\log n)^{2}\right\rceil$. Note that, for $n$ large enough, $n>3 k_{n}$. We set

$$
A_{m, n}(u, v):=\left(\mathbb{E}_{\bar{\mu}}\left[u \cdot e^{i t * S_{n}} \cdot v \circ \bar{T}^{n}\right] / \lambda_{t}^{n}\right)_{\mid t=0}^{(m)} .
$$

Note that, for every integers $k, n>0$,

$$
\begin{aligned}
\mid A_{m, n}(u, v)-A_{m, n}\left(u_{k}, v_{k}\right) \| & \leq\left\|\frac{\partial^{m}}{\partial t^{m}}\left(\frac{e^{i t * S_{n}}}{\lambda_{t}^{n}}\right)_{\mid t=0}\right\|_{L^{1}(\bar{\mu})}\|u\|_{(\xi)}\|v\|_{(\xi)} \xi^{k} \\
& \leq \tilde{c}_{m} n^{m}\|u\|_{(\xi)}\|v\|_{(\xi)} \xi^{k} .
\end{aligned}
$$

For every integers $n, n^{\prime}$ such that $0<n \leq n^{\prime} \leq 2 n$, we have

$$
\begin{aligned}
& \left|A_{m, n}(u, v)-A_{m, n^{\prime}}(u, v)\right| \\
\leq & \left|A_{m, n}\left(u_{k_{n}}, v_{k_{n}}\right)-A_{m, n^{\prime}}\left(u_{k_{n}}, v_{k_{n}}\right)\right|+\left(1+2^{m}\right) \tilde{c}_{m} n^{m}\|u\|_{(\xi)}\|v\|_{(\xi)} \xi^{k_{n}} \\
\leq & K_{m}\|u\|_{(\xi)}\|v\|_{(\xi)} \xi_{0}^{k_{n}},
\end{aligned}
$$

due to (25). Hence, we conclude that $\left(A_{m, n}(u, v)\right)_{n}$ is a Cauchy sequence so that $A_{m}(u, v)$ is well defined and that

$$
\left|A_{m}(u, v)-A_{m, n}(u, v)\right| \leq K_{m}\|u\|_{(\xi)}\|v\|_{(\xi)} \sum_{j \geq 0} \xi_{0}^{k_{2 j}}=O\left(\|u\|_{(\xi)}\|u\|_{(\xi)} \xi_{0}^{k_{n}}\right) .
$$

Since Applying Proposition 4.2 to the couple $\left(u_{\left(k_{n}\right)}, v_{\left(k_{n}\right)}\right)$ leads to (33).

\subsection{Proofs of our main results.}

Theorem 4.5. Let $f, g: M \rightarrow \mathbb{R}$ be two bounded observables such that

$$
\sum_{\ell \in \mathbb{Z}^{2}}\left(\left\|f \mathbf{1}_{\mathcal{C}_{\ell}}\right\|_{(\xi)}+\left\|g \mathbf{1}_{\mathcal{C}_{\ell}}\right\|_{(\xi)}\right)<\infty
$$

Then

$$
\begin{aligned}
& \int_{M} f . g \circ T^{n} d \nu \\
= & \left.\sum_{m=0}^{2 K-2} \frac{1}{m !} \sum_{j=0}^{2 K-2-m} \frac{i^{m+2 j}}{(2 j) !} \frac{\sum_{\ell, \ell^{\prime} \in \mathbb{Z}^{2}} \Phi^{(m+2 j)}\left(\frac{\ell^{\prime}-\ell}{\sqrt{n}}\right) *\left(A_{m}\left(u_{\ell}, v_{\ell^{\prime}}\right)\right)}{n^{j+1+\frac{m}{2}}} *\left(\lambda^{n} / a^{n}\right)_{0}^{(2 j)}\right)+o\left(n^{-K}(35)\right.
\end{aligned}
$$

with $u_{\ell}(q, \vec{v})=f(q+\ell, \vec{v})$ and $v_{\ell}(q, \vec{v})=f(q+\ell, \vec{v})$ and with $A_{m}(u, v)$ given by (34).

If moreover, $\sum_{\ell \in \mathbb{Z}^{2}}|\ell|^{2 K-2}\left(\left\|f \mathbf{1}_{\mathcal{C}_{\ell}}\right\|_{(\xi)}+\left\|g \mathbf{1}_{\mathcal{C}_{\ell}}\right\|_{(\xi)}\right)<\infty$, then

$$
\int_{M} f . g \circ T^{n} d \nu=\sum_{L=0}^{K-1} \frac{\tilde{c}_{L}}{n^{1+L}} \sum_{j=0}^{2 K-2-2 L}(-1)^{j} \frac{\Phi^{(2 j+2 L)}(0)}{(2 j) ! n^{j}} *\left(\lambda^{n} / a^{n}\right)_{0}^{(2 j)}+o\left(n^{-K}\right)
$$


with

$$
\tilde{c}_{L}(f, g):=\sum_{r, m \geq 0} \frac{i^{m}}{m ! r !} \sum_{\ell, \ell^{\prime} \in \mathbb{Z}^{2}}\left(\ell^{\prime}-\ell\right)^{\otimes r} \otimes A_{m}\left(u_{\ell}, v_{\ell^{\prime}}\right) .
$$

Since $\left(\lambda^{n} / a^{n}\right)_{0}^{(2 j)}=O\left(n^{j / 2}\right)$, we conclude that:

Remark 4.6. Assume $\sum_{\ell \in \mathbb{Z}^{2}}|\ell|^{2 K-2}\left(\left\|f \mathbf{1}_{\mathcal{C}_{\ell}}\right\|_{(\xi)}+\left\|g \mathbf{1}_{\mathcal{C}_{\ell}}\right\|_{(\xi)}\right)<\infty$ and $\int_{M} f . g d \nu=O\left(n^{-K}\right)$. Then

$$
\int_{M} f . g d \nu=\frac{\Phi^{(2 K-2)}(0) * \tilde{c}_{K-1}(f, g)}{n^{K}}+o\left(n^{-K}\right)
$$

and $\tilde{c}_{K-1}(f, g)=\lim _{n \rightarrow+\infty} \frac{(-1)^{K-1}}{(2 K-2) !} \sum_{\ell, \ell^{\prime} \in \mathbb{Z}^{2}} \mathbb{E}_{\bar{\mu}}\left[u_{\ell} \cdot \frac{\partial^{2 K-2}}{\partial t^{2 K-2}}\left(\lambda_{t}^{-n} e^{i t *\left(S_{n}-\left(\ell^{\prime}-\ell\right)\right)}\right)_{\mid t=0} \cdot v_{\ell^{\prime}} \circ \bar{T}^{n}\right]$.

Corollary 4.7. Under the assumptions of Theorem 4.5 ensuring (36), using the fact that $(\lambda / a)_{0}^{(2 j)}=O\left(n^{j / 2}\right)$, as in Remarks [2.6 and 4.3, if $\sum_{\ell \in \mathbb{Z}^{2}}|\ell|^{4 K-4}\left(\left\|f \mathbf{1}_{\mathcal{C}_{\ell}}\right\|_{(\xi)}+\left\|g \mathbf{1}_{\mathcal{C}_{\ell}}\right\|_{(\xi)}\right)<\infty$, the right hand side of (36) can be rewritten

$$
n^{-\frac{d}{2}} \sum_{\ell, \ell^{\prime} \in \mathbb{Z}^{2}} \sum_{L=0}^{4 K-4} \frac{\Phi^{(L)}(0)}{L !} i^{L} \frac{\partial^{L}}{\partial t^{L}}\left(\mathbb{E}_{\bar{\mu}}\left[u_{\ell} \cdot e^{i t * \frac{\left(S_{n}-\left(\ell^{\prime}-\ell\right)\right)}{\sqrt{n}}} \cdot v_{\ell^{\prime}} \circ \bar{T}^{n}\right] e^{\frac{1}{2} \Sigma^{2} * t^{\otimes 2}}\right)_{\mid t=0}+o\left(n^{-K}\right) .
$$

Proof of Theorem 4.5. We have

$$
\int_{M} f . g \circ T^{n} d \nu=\sum_{\ell, \ell^{\prime} \in \mathbb{Z}^{2}} \mathbb{E}_{\bar{\mu}}\left[u_{\ell} \mathbf{1}_{\left\{S_{n}=\ell^{\prime}-\ell\right\}} v_{\ell^{\prime}} \circ \bar{T}^{n}\right] .
$$

Hence, (35) follows directly from Proposition 4.4. Due to the dominated convergence theorem,

$$
\begin{aligned}
& \lim _{n \rightarrow+\infty} n^{K-1-\frac{m+j}{2}} \sum_{\ell, \ell^{\prime} \in \mathbb{Z}^{2}}\left(\Phi^{(m+j)}\left(\frac{\ell^{\prime}-\ell}{\sqrt{n}}\right)-\sum_{r=0}^{2 K-2-m-(j / 2)} \frac{\Phi^{(m+j+r)}(0)}{r !} *\left(\frac{\ell^{\prime}-\ell}{\sqrt{n}}\right)^{\otimes r}\right) \\
& *\left(\left(\lambda^{n} / a^{n}\right)_{0}^{(j)} \otimes A_{m}\left(u_{\ell}, v_{\ell^{\prime}}\right)\right)=0,
\end{aligned}
$$

(where we used (26) ) and to the fact that the uneven derivatives of $\Phi$ are null and that $\Phi^{(2 k)}(0)=$ $\left(-\Sigma^{2}\right)^{\otimes k} \Phi(0)$. Therefore

$$
\begin{aligned}
\int_{M} f . g \circ T^{n} d \nu= & \sum_{m=0}^{2 K-2} \sum_{r=0: r+m \in 2 \mathbb{Z}}^{2 K-2-m} \frac{\Phi(0)}{m ! r !} \sum_{j=0}^{2 K-2-m-r} \frac{(-1)^{j}}{(2 j) !}\left(\frac{\left(-\Sigma^{-2}\right)^{\otimes\left(j+\frac{m+r}{2}\right)}}{n^{j+1+\frac{m+r}{2}}} *\left(\lambda^{n} / a^{n}\right)_{0}^{(2 j)}\right) \\
& * \sum_{\ell, \ell^{\prime} \in \mathbb{Z}^{2}} i^{m}\left(\ell^{\prime}-\ell\right)^{\otimes r} \otimes A_{m}\left(u_{\ell}, v_{\ell^{\prime}}\right)+o\left(n^{-K}\right),
\end{aligned}
$$

which ends the proof of (36).

Proof of Theorem 1.2. This comes from (36) combined with the fact that $\left(\lambda^{n} / a^{n}\right)_{0}^{(2 j)}$ is a polynomial in $n$ of degree bounded by $j / 2$.

Proof of Theorem 1.3. Due to (36) of Theorem 4.5, we obtain (7) with

$$
\tilde{\mathfrak{A}}_{2}(f, g)=\mathfrak{a}_{2,0,0}(f, g)+\mathfrak{a}_{0,2,0}(f, g)+\mathfrak{a}_{1,1,0}(f, g),
$$


where $a_{m, r, j}(f, g)$ corresponds to the contribution of the $(m, r, j)$-term in the sum of the right hand side of (36). Moreover, due to Proposition A.2.

$$
\begin{aligned}
\mathfrak{a}_{2,0,0}(f, g)= & \sum_{\ell, \ell^{\prime} \in \mathbb{Z}^{2}} A_{2}\left(u_{\ell}, v_{\ell^{\prime}}\right) \\
= & -\lim _{n \rightarrow+\infty}\left\{\int_{M} f d \nu \sum_{j, m=-n}^{-1} \int_{M} g \cdot\left(\kappa \circ T^{j} \otimes \kappa \circ T^{m}-\mathbb{E}_{\bar{\mu}}\left[\kappa \circ \bar{T}^{j} \otimes \kappa \circ \bar{T}^{m}\right]\right)\right] d \nu \\
& +\int_{M} g d \nu \sum_{j, m=0}^{n-1} \int_{M} f \cdot\left[\kappa \circ T^{j} \otimes \kappa \circ T^{m}-\mathbb{E}_{\bar{\mu}}\left[\kappa \circ T^{j} \otimes \kappa \circ T^{m}\right]\right] d \nu \\
& +2 \sum_{r=0}^{n-1} \int_{M} f \cdot \kappa \circ T^{r} d \nu \otimes \sum_{m=-n}^{-1} \int_{M} g \cdot \kappa \circ T^{m} d \nu \\
& \left.+\int_{M} f d \nu \int_{M} g d \nu\left(\mathbb{E}_{\bar{\mu}}\left[S_{n}^{\otimes 2}\right]-n \Sigma^{2}\right)\right\}, \\
\mathfrak{a}_{0,2,0}(f, g)= & -\sum_{\ell, \ell^{\prime} \in \mathbb{Z}^{2}} A_{0}\left(u_{\ell}, v_{\ell^{\prime}}\right) \cdot\left(\ell^{\prime}-\ell\right)^{\otimes 2}=-\sum_{\ell, \ell^{\prime} \in \mathbb{Z}^{2}}\left(\ell^{\prime}-\ell\right)^{\otimes 2} \int_{\mathcal{C}_{\ell}} f d \nu \int_{\mathcal{C}_{\ell^{\prime}}} g d \nu \\
\mathfrak{a}_{1,1,0}(f, g) & -2 i \sum_{\ell, \ell^{\prime} \in \mathbb{Z}^{2}} A_{1}\left(u_{\ell}, v_{\ell^{\prime}}\right) \otimes\left(\ell^{\prime}-\ell\right) \\
& =\sum_{n \rightarrow+\infty} \sum_{\ell, \ell^{\prime} \in \mathbb{Z}^{2}} \int_{\mathcal{C}_{\ell^{\prime}}} g d \nu \sum_{r=0}^{n-1} \int_{\mathcal{C}_{\ell}} f .\left(\left(\ell^{\prime}-\ell\right) \otimes \kappa \circ T^{r}\right) d \nu \\
& \left.+\sum_{\mathcal{C}_{\ell}} f d \nu \sum_{m=-n} \int_{\mathcal{C}_{\ell^{\prime}}} g \cdot\left(\left(\ell^{\prime}-\ell\right) \otimes \kappa \circ T^{m}\right) d \nu\right\} .
\end{aligned}
$$

For the contribution of the term with $(m, r, j)=(0,0,2)$, note that

$$
\left(\lambda^{n} / a^{n}\right)_{0}^{(4)}=n(\lambda / a)_{0}^{(4)}=n\left(\lambda_{0}^{(4)}-3\left(\Sigma^{2}\right)^{\otimes 2}\right) .
$$

Moreover, due to Proposition A.3,

$$
\lambda_{0}^{(4)}-3\left(\Sigma^{2}\right)^{\otimes 2}=\lim _{n \rightarrow+\infty} \frac{\mathbb{E}_{\bar{\mu}}\left[S_{n}^{\otimes 4}\right]-3 n^{2}\left(\Sigma^{2}\right)^{\otimes 2}}{n}+6 \Sigma^{2} \otimes B_{0}=\Lambda_{4} .
$$

Note that

$$
\begin{aligned}
& \mathfrak{a}_{2,0,0}(f, g)=-\lim _{n \rightarrow+\infty}\left\{\int_{M} f d \nu \int_{M} g\left(\left(\mathcal{I}_{0}-\mathcal{I}_{-n}\right)^{\otimes 2}-\mathbb{E}_{\bar{\mu}}\left[S_{n}^{\otimes 2}\right]\right) d \nu\right. \\
&+\int_{M} g d \nu \int_{M} f\left(\left(\mathcal{I}_{n}-\mathcal{I}_{0}\right)^{\otimes 2}-\mathbb{E}_{\bar{\mu}}\left[S_{n}^{\otimes 2}\right]\right) d \nu \\
&+2 \int_{M} f\left(\mathcal{I}_{n}-\mathcal{I}_{0}\right) d \nu \otimes \int_{M} g\left(\mathcal{I}_{0}-\mathcal{I}_{-n}\right) d \nu \\
&\left.-\int_{M} f d \nu \int_{M} g d \nu \mathfrak{B}_{0}\right\}, \\
& \mathfrak{a}_{0,2,0}(f, g)=-\int_{M} f \cdot \mathcal{I}_{0}^{\otimes 2} d \nu \int_{M} g d \nu-\int_{M} f d \nu \int_{M} g \cdot \mathcal{I}_{0}^{\otimes 2} d \nu+2 \int_{M} f \mathcal{I}_{0} d \nu \otimes \int_{M} g \mathcal{I}_{0} d \nu
\end{aligned}
$$


and

$$
\begin{aligned}
\mathfrak{a}_{1,1,0}(f, g)= & \lim _{n \rightarrow+\infty}\left\{2 \int_{M} g \mathcal{I}_{0} d \nu \otimes \int_{M} f\left(\mathcal{I}_{n}-\mathcal{I}_{0}\right) d \nu-2 \int_{M} g d \nu \int_{M} f \cdot \mathcal{I}_{0} \otimes\left(\mathcal{I}_{n}-\mathcal{I}_{0}\right) d \nu\right. \\
& \left.+2 \int_{M} f d \nu \int_{M} g \cdot \mathcal{I}_{0} \otimes\left(\mathcal{I}_{0}-\mathcal{I}_{-n}\right) d \nu-2 \int_{M} f \mathcal{I}_{0} d \nu \otimes \int_{M} g\left(\mathcal{I}_{0}-\mathcal{I}_{-n}\right) d \nu\right\} .
\end{aligned}
$$

Hence we have proved (7) with

$$
\tilde{\mathfrak{A}}_{2}(f, g):=-\int_{M} f d \nu \tilde{\mathfrak{B}}_{2}^{-}(g)-\int_{M} g d \nu \tilde{\mathfrak{B}}_{2}^{+}(f)+\int_{M} f d \nu \int_{M} g d \nu \mathfrak{B}_{0}+2 \mathfrak{B}_{1}^{+}(f) \otimes \mathfrak{B}_{1}^{-}(g),
$$

with

$$
\begin{aligned}
\tilde{\mathfrak{B}}_{2}^{+}(f) & :=\lim _{m \rightarrow+\infty} \int_{M} f\left(\mathcal{I}_{m}^{\otimes 2}-\mathbb{E}\left[S_{m}^{\otimes 2}\right]\right) d \nu, \\
\mathfrak{B}_{2}^{-}(g) & :=\lim _{m \rightarrow-\infty} \int_{M} g\left(\mathcal{I}_{m}^{\otimes 2}-\mathbb{E}\left[S_{m}^{\otimes 2}\right]\right) d \nu .
\end{aligned}
$$

Remark 4.8. Let $f, g: M \rightarrow \mathbb{R}$ be two bounded observables such that

$$
\sum_{\ell \in \mathbb{Z}^{2}}|\ell|^{4}\left(\left\|f \mathbf{1}_{\mathcal{C}_{\ell}}\right\|_{(\xi)}+\left\|g \mathbf{1}_{\mathcal{C}_{\ell}}\right\|_{(\xi)}\right)<\infty
$$

Assume moreover that $\int_{M} f d \nu \int_{M} g d \nu=0$ and that $\tilde{\mathfrak{A}}_{2}(f, g)=0$. Due to Remark 4.6 ,

$$
\begin{aligned}
& \int_{M} f \cdot g \circ T^{n} d \nu \\
& =\frac{\left(\Sigma^{-2}\right)^{\otimes 2}}{2 \pi \sqrt{\operatorname{det} \Sigma^{2}} n^{3}} * \sum_{\ell, \ell^{\prime} \in \mathbb{Z}^{2}}\left(\frac{A_{4}\left(u_{\ell}, v_{\ell^{\prime}}\right)}{24}+\frac{A_{0}\left(u_{\ell}, v_{\ell^{\prime}}\right)}{24}\left(\ell^{\prime}-\ell\right)^{\otimes 4}+\frac{i A_{1}\left(u_{\ell}, v_{\ell^{\prime}}\right)}{6} \otimes\left(\ell^{\prime}-\ell\right)^{\otimes 3}\right. \\
& \left.-\frac{1}{4} A_{2}\left(u_{\ell}, v_{\ell^{\prime}}\right) \otimes\left(\ell^{\prime}-\ell\right)^{\otimes 2}-\frac{i}{6} A_{3}\left(u_{\ell}, v_{\ell^{\prime}}\right) \otimes\left(\ell^{\prime}-\ell\right)\right)+o\left(n^{-3}\right)
\end{aligned}
$$

where $u_{\ell}(q, \vec{v}):=f(q+\ell, \vec{v})$ and $v_{\ell}(q, \vec{v}):=g(q+\ell, \vec{v})$.

Proof of Proposition 1.6. We apply Remark 4.8, Using the definitions of $A_{0}$ and $A_{1}$, we observe that

$$
\forall \ell, \ell^{\prime} \in \mathbb{Z}^{2}, \quad A_{0}\left(u_{\ell}, v_{\ell^{\prime}}\right)=A_{1}\left(u_{\ell}, v_{\ell^{\prime}}\right)=0
$$

(since $\mathbb{E}_{\bar{\mu}}\left[u_{\ell}\right]=\mathbb{E}_{\bar{\mu}}\left[v_{\ell^{\prime}}\right]=0$ ) and

$$
\sum_{\ell, \ell^{\prime} \in \mathbb{Z}^{2}} A_{4}\left(u_{\ell}, v_{\ell^{\prime}}\right)=A_{4}\left(\sum_{\ell \in \mathbb{Z}^{2}} u_{\ell}, \sum_{\ell^{\prime} \in \mathbb{Z}^{2}} v_{\ell^{\prime}}\right)=0 .
$$

Moreover

$$
\sum_{\ell, \ell^{\prime} \in \mathbb{Z}^{2}} A_{3}\left(u_{\ell}, v_{\ell^{\prime}}\right) \otimes\left(\ell^{\prime}-\ell\right)=\sum_{\ell, \ell^{\prime} \in \mathbb{Z}^{2}} h_{\ell} q_{\ell^{\prime}} A_{3}\left(f_{0}, g_{0}\right) \otimes\left(\ell^{\prime}-\ell\right)=0
$$


since $\sum_{\ell \in \mathbb{Z}^{2}} h_{\ell}=\sum_{\ell} q_{\ell}=0$. Therefore

$$
\begin{aligned}
& \int_{M} f . g \circ T^{n} d \nu \\
& =-\frac{1}{4} \frac{\left(\Sigma^{-2}\right)^{\otimes 2}}{2 \pi \sqrt{\operatorname{det} \Sigma^{2}} n^{3}} * \sum_{\ell, \ell^{\prime} \in \mathbb{Z}^{2}} A_{2}\left(u_{\ell}, v_{\ell^{\prime}}\right) \otimes\left(\ell^{\prime}-\ell\right)^{\otimes 2}+o\left(n^{-3}\right) \\
& =\frac{1}{2} \frac{\left(\Sigma^{-2}\right)^{\otimes 2}}{2 \pi \sqrt{\operatorname{det} \Sigma^{2}} n^{3}} * \sum_{\ell, \ell^{\prime} \in \mathbb{Z}^{2}} h_{\ell} q_{\ell^{\prime}} A_{2}\left(f_{0}, g_{0}\right) \otimes \ell \otimes \ell^{\prime}+o\left(n^{-3}\right) \\
& =\frac{1}{2} \frac{\left(\Sigma^{-2}\right)^{\otimes 2}}{2 \pi \sqrt{\operatorname{det} \Sigma^{2}} n^{3}} A_{2}\left(f_{0}, g_{0}\right) \otimes \sum_{\ell \in \mathbb{Z}^{2}} h_{\ell} \cdot \ell \otimes \sum_{\ell^{\prime} \in \mathbb{Z}^{2}} q_{\ell^{\prime}} \cdot \ell^{\prime}+o\left(n^{-3}\right) \\
& =-\frac{\left(\Sigma^{-2}\right)^{\otimes 2}}{2 \pi \sqrt{\operatorname{det} \Sigma^{2}} n^{3}} *\left(\sum_{j \geq 0} \mathbb{E}_{\bar{\mu}}\left[f_{0} \cdot \kappa \circ \bar{T}^{j}\right] \otimes \sum_{m \leq-1} \mathbb{E}_{\bar{\mu}}\left[g_{0} \cdot \kappa \circ \bar{T}^{m}\right] \otimes \sum_{\ell \in \mathbb{Z}^{2}} h_{\ell} \cdot \ell \otimes \sum_{\ell^{\prime} \in \mathbb{Z}^{2}} q_{\ell^{\prime} \cdot \ell^{\prime}}\right)+o\left(n^{-3}\right) \\
& =-\frac{\left(\Sigma^{-2}\right)^{\otimes 2}}{2 \pi \sqrt{\operatorname{det} \Sigma^{2}} n^{3}} *\left(\sum_{j \geq 0} \int_{M} f \mathcal{I}_{0} \otimes \kappa \circ T^{j} d \nu \otimes \sum_{m \leq-1} \int_{M} g \mathcal{I}_{0} \otimes \kappa \circ T^{m} d \nu\right)+o\left(n^{-3}\right) .
\end{aligned}
$$

\section{Proof of the MiXing RESUlt in the INFINITE HORIZON CASE}

Proof of Theorem 1.1. In 28, Szász and Varjú implemented the Nagaev-Guivarc'h perturbation method via the Keller-Liverani theorem [12 to prove that Hypothesis 2.1 holds true for the dynamical system $(\hat{M}, \hat{\mu}, \hat{T})$ with the Young Banach space $\mathcal{B}$, with $\mathcal{B}_{0}:=\mathbb{L}^{1}(\hat{\mu})$ and with $\lambda$ having the following expansion:

$$
\lambda_{t}-1 \sim \Sigma_{\infty}^{2} *\left(t^{\otimes 2}\right) \log |t| .
$$

Hence Hypothesis 2.2 holds also true, with $\Theta_{n}=\sqrt{n \log n} I d$ and with $Y$ a gaussian random variable with distribution $\mathcal{N}\left(0, \Sigma_{\infty}^{2}\right)$ with density function $\Phi(x)=\exp \left(-\frac{1}{2}\left(\Sigma_{\infty}^{2}\right)^{-1} * x^{\otimes 2}\right) /\left(2 \pi \sqrt{\operatorname{det} \Sigma_{\infty}^{2}}\right)$. Let $k_{n}:=\left\lceil\log ^{2} n\right\rceil$. Let $u_{n}(x)$ and $v_{n}(x)$ correspond to the conditional expectation of respectively $f$ and $g$ over the connected component of $M \backslash \bigcup_{m=-k_{n}}^{k_{n}} T^{-m} \mathcal{S}_{0}$ containing $x$. First note that

$$
\int_{M} f . g \circ T^{n} d \nu=\int_{M} u_{n} \cdot v_{n} \circ T^{n} d \nu+O\left(\left(L_{\xi}(f) \int_{M}|g| d \nu+L_{\xi}(g) \int_{M}|f| d \nu\right) \xi^{k_{n}}\right) .
$$

As noticed in Proposition 3.2, there exist $\hat{f}_{n}, \hat{g}_{n}: \hat{M} \times \mathbb{Z}^{2} \rightarrow \mathbb{C}$ such that

$$
\begin{array}{ll}
\forall \tilde{x} \in \tilde{M}, & \hat{f}_{n}(\hat{\pi}(\tilde{x}), \ell)=u_{n}\left(\bar{T}^{k_{n}}(\tilde{\pi}(\tilde{x}))+\ell\right), \\
\forall \tilde{x} \in \tilde{M}, \quad \hat{g}_{n}(\hat{\pi}(\tilde{x}), \ell)=v_{n}\left(\bar{T}^{k_{n}}(\tilde{\pi}(\tilde{x}))+\ell\right),
\end{array}
$$

with the notation $(q, \vec{v})+\ell=(q+\ell, \vec{v})$ for every $(q, \vec{v}) \in \bar{M}$. For $n$ large enough, $n>3 k_{n}$ and, due to (23),

$$
\begin{aligned}
& \int_{M} u_{n} \cdot v_{n} \circ T^{n} d \nu=\sum_{\ell, \ell^{\prime} \in \mathbb{Z}^{2}} \mathbb{E}_{\bar{\mu}}\left[u_{n}(\cdot+\ell) \cdot \mathbf{1}_{S_{n}=\ell^{\prime}-\ell \cdot v_{n}}\left(\bar{T}^{n}(\cdot)+\ell^{\prime}\right)\right] \\
& =\sum_{\ell, \ell^{\prime} \in \mathbb{Z}^{2}} \frac{1}{(2 \pi)^{2}} \int_{[-\pi, \pi]^{2}} e^{-i t *\left(\ell^{\prime}-\ell\right)} \mathbb{E}_{\bar{\mu}}\left[u_{n}(\cdot+\ell) \cdot e^{i t * S_{n}} \cdot v_{n}\left(\bar{T}^{n}(\cdot)+\ell^{\prime}\right)\right] \\
& =\sum_{\ell, \ell^{\prime} \in \mathbb{Z}^{2}} \frac{1}{(2 \pi)^{2}} \int_{[-\pi, \pi]^{2}} e^{-i t *\left(\ell^{\prime}-\ell\right)} \mathbb{E}_{\hat{\mu}}\left[\hat{G}_{n, t}\left(\cdot, \ell^{\prime}\right) \hat{P}_{t}^{n-2 k_{n}} \hat{P}^{2 k_{n}}\left(\hat{F}_{n, t}(\cdot, \ell)\right)\right] d t
\end{aligned}
$$


where $\hat{F}_{n, t}, \hat{G}_{n, t}: \hat{M} \rightarrow \mathbb{Z}^{2} \rightarrow \mathbb{C}$ are the functions defined by

$$
\begin{gathered}
\hat{F}_{n, t}(\hat{x}, \ell):=\hat{f}_{n}(\hat{x}, \ell) \cdot e^{i t * \hat{S}_{k_{n}}\left(\hat{T}^{k_{n}}(\hat{x})\right)}, \\
\hat{G}_{n, t}(\hat{x}, \ell):=\hat{g}_{n}(\hat{x}, \ell) \cdot e^{i t * \hat{S}_{k_{n}}(\hat{x})} .
\end{gathered}
$$

Moreover $\sup _{n, t}\left\|\hat{P}^{2 k_{n}} \hat{F}_{n, t}(\cdot, \ell)\right\| \leq\left(1+2 \beta^{-1}\right)\left\|f \mathbf{1}_{\mathcal{C}_{\ell}}\right\|_{\infty}$. Hence, due to Hypothesis 2.1 ,

$$
\begin{aligned}
& \int_{M} u_{n} \cdot v_{n} \circ T^{n} d \nu \\
& =O\left(\vartheta^{n-2 k_{n}}\right)+\sum_{\ell, \ell^{\prime} \in \mathbb{Z}^{2}} \frac{1}{(2 \pi)^{2}} \int_{[-\pi, \pi]^{2}} e^{-i t *\left(\ell^{\prime}-\ell\right)} \mathbb{E}_{\hat{\mu}}\left[\hat{G}_{n, t}\left(\cdot, \ell^{\prime}\right) \lambda_{t}^{n-2 k_{n}} \Pi_{t} \hat{P}^{2 k_{n}}\left(\hat{F}_{n, t}(\cdot, \ell)\right)\right] d t \\
& =O\left(\vartheta^{n-2 k_{n}}\right)+\sum_{\ell, \ell^{\prime} \in \mathbb{Z}^{2}} \frac{1}{\mathfrak{a}_{n}^{2}(2 \pi)^{2}} \int_{\left[-\mathfrak{a}_{n} \pi, \mathfrak{a}_{n} \pi\right]^{2}} e^{-i u * \frac{\ell^{\prime}-\ell}{\mathfrak{a}_{n}}} \mathbb{E}_{\hat{\mu}}\left[\hat{G}_{n, u / \mathfrak{a}_{n}}\left(\cdot, \ell^{\prime}\right) \lambda_{u / \mathfrak{a}_{n}}^{n-2 k_{n}} \Pi_{u / \mathfrak{a}_{n}} \hat{P}^{2 k_{n}}\left(\hat{F}_{n, u / \mathfrak{a}_{n}}(\cdot, \ell)\right)\right] d u
\end{aligned}
$$

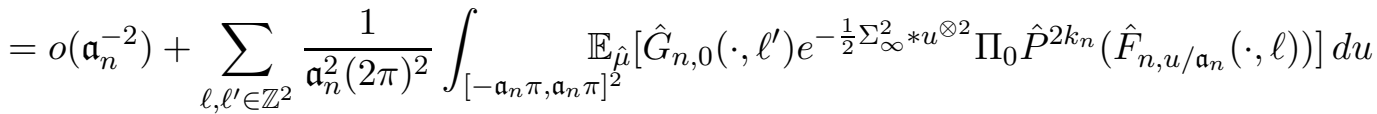

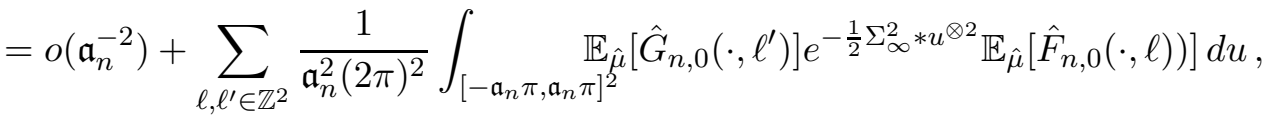

where we used the change of variable $u=\mathfrak{a}_{n} t$ with $\mathfrak{a}_{n}:=\sqrt{\left(n-2 k_{n}\right) \log \left(n-2 k_{n}\right)}$, and twice the dominated convergence theorem. Therefore

$$
\int_{M} u_{n} \cdot v_{n} \circ T^{n} d \nu=\frac{\Phi(0)}{\mathfrak{a}_{n}^{2}(2 \pi)^{2}} \int_{M} u_{n} d \nu \int_{M} v_{n} d \nu+o\left(\mathfrak{a}_{n}^{-2}\right) .
$$

The conclusion of the theorem follows from this last formula combined with (38) and with the facts that $\mathfrak{a}_{n}^{2} \sim n \log n$ and that

$$
\int_{M} u_{n} d \nu \int_{M} v_{n} d \nu=\int_{M} f d \nu \int_{M} g d \nu
$$

due to the dominated convergence theorem.

\section{Appendix A. Billiard with finite horizon: about the Coefficients $A_{m}$}

Let $\mathcal{W}^{s}$ (resp. $\mathcal{W}^{u}$ ) be the set of stable (resp. unstable) $H$-manifolds. In [6], Chernov defines two separation times $s_{+}$and $s_{-}$which are dominated by $s$ and such that, for every positive integer $k$,

$$
\begin{gathered}
\forall W^{u} \in \mathcal{W}^{u}, \forall \bar{x}, \bar{y} \in W^{u}, \quad s^{+}\left(\bar{T}^{-k} \bar{x}, \bar{T}^{-k} \bar{y}\right)=s^{+}(x, y)+k, \\
\forall W^{s} \in \mathcal{W}^{s}, \forall \bar{x}, \bar{y} \in W^{s}, \quad s^{-}\left(\bar{T}^{k} \bar{x}, \bar{T}^{k} \bar{y}\right)=s^{-}(x, y)+k .
\end{gathered}
$$

Proposition A.1 ([6], Theorem 4.3 and remark after). There exist $C_{0}>0$ and $\vartheta_{0} \in(0,1)$ such that, for every positive integer $n$, for every bounded measurable $u, v: \bar{M} \rightarrow \mathbb{R}$,

$$
\mid \mathbb{E}_{\bar{\mu}}\left[u . v \circ \bar{T}^{n}\right]-\mathbb{E}_{\bar{\mu}}[u] \mathbb{E}_{\bar{\mu}}[v] \| \leq C_{0}\left(L_{u}^{+}\|v\|_{\infty}+L_{v}^{-}\|u\|_{\infty}+\|u\|_{\infty}\|v\|_{\infty}\right) \vartheta_{0}^{n},
$$

with

$$
L_{u}^{+}:=\sup _{W^{u} \in \mathcal{W}^{u}} \sup _{x, y \in W^{u}, x \neq y}\left(|u(x)-u(y)| \xi^{-\mathbf{s}_{+}(x, y)}\right),
$$

and

$$
L_{v}^{-}:=\sup _{W^{s} \in \mathcal{W}^{s}} \sup _{x, y \in W^{s}, x \neq y}\left(|v(x)-v(y)| \xi^{-\mathbf{s}_{-}(x, y)}\right) .
$$


Note that

$$
\begin{gathered}
L_{u}^{+} \leq L_{\xi}\left(u \mathbf{1}_{\bar{M}}\right), \quad L_{u}^{-} \leq L_{\xi}\left(u \mathbf{1}_{\bar{M}}\right), \\
L_{u \circ \bar{T}^{-k}}^{+} \leq L_{u}^{+} \xi^{k} \quad \text { and } \quad L_{v \circ \bar{T}^{k}}^{-} \leq L_{v}^{-} \xi^{k} .
\end{gathered}
$$

We will set $\tilde{u}:=u-\mathbb{E}_{\bar{\mu}}[u]$ and $\tilde{v}:=v-\mathbb{E}_{\bar{\mu}}[v]$. We will express the terms $A_{m}(u, v)$ for $m \in\{1,2,3,4\}$ in terms of the follwing quantities:

$$
\begin{aligned}
& B_{1}^{+}(u):=\sum_{j \geq 0} \mathbb{E}_{\bar{\mu}}\left[u . \kappa \circ T^{j}\right], \quad B_{1}^{-}(v):=\sum_{m \leq-1} \mathbb{E}_{\bar{\mu}}\left[v . \kappa \circ \bar{T}^{m}\right], \\
& B_{2}^{+}(u):=\sum_{j, m \geq 0} \mathbb{E}_{\bar{\mu}}\left[\tilde{u} . \kappa \circ \bar{T}^{j} \otimes \kappa \circ \bar{T}^{m}\right], \quad B_{2}^{-}(v):=\sum_{j, m \leq-1} \mathbb{E}_{\bar{\mu}}\left[\tilde{v} \cdot \kappa \circ \bar{T}^{j} \otimes \kappa \circ \bar{T}^{m}\right], \\
& B_{0}^{-}(v):=\sum_{k \leq-1}|k| \mathbb{E}_{\bar{\mu}}\left[\tilde{v} . \kappa \circ \bar{T}^{k}\right], \quad B_{0}^{+}(u)=\sum_{k \geq 0} k \mathbb{E}_{\bar{\mu}}\left[\tilde{u} . \kappa \circ \bar{T}^{k}\right], \\
& B_{0}:=B_{0}^{-}(\kappa)+B_{0}^{+}(\kappa)=\sum_{m \in \mathbb{Z}}|m| \mathbb{E}_{\bar{\mu}}\left[\kappa \otimes \kappa \circ \bar{T}^{m}\right], \\
& B_{0,2}^{+}(u):=\sum_{k, m \geq 0} \max (k, m) \mathbb{E}_{\bar{\mu}}\left[\tilde{u} . \kappa \circ \bar{T}^{k} \otimes \kappa \circ \bar{T}^{m}\right] . \\
& B_{0,2}^{-}(v):=\sum_{k, m \geq 1} \max (k, m) \mathbb{E}_{\bar{\mu}}\left[\tilde{v} . \kappa \circ \bar{T}^{-k} \otimes \kappa \circ \bar{T}^{-m}\right], \\
& B_{3}^{+}(u):=\sum_{k, r, m \geq 0} \mathbb{E}_{\bar{\mu}}\left[\tilde{u} . \kappa \circ \bar{T}^{\min (k, r, m)}\right. \\
& \left.\left(\kappa \circ \bar{T}^{\max (k, r, m)} \otimes \kappa \circ \bar{T}^{\operatorname{med}(k, r, m)}-\mathbb{E}_{\bar{\mu}}\left[\kappa \circ \bar{T}^{\max (k, r, m)} \otimes \kappa \circ \bar{T}^{\operatorname{med}(k, r, m)}\right]\right)\right], \\
& B_{3}^{-}(v):=\sum_{m, r, s \leq-1} \mathbb{E}_{\bar{\mu}}\left[\tilde{v} \cdot \kappa \circ \bar{T}^{\max (m, r, s)} \otimes\right. \\
& \left.\left(\kappa \circ \bar{T}^{\min (m, r, s)} \otimes \kappa \circ \bar{T}^{m e d(m, r, s)}-\mathbb{E}_{\bar{\mu}}\left[\kappa \circ \bar{T}^{\min (m, r, s)} \otimes \kappa \circ \bar{T}^{m e d(m, r, s)}\right]\right)\right],
\end{aligned}
$$

with $\operatorname{med}(m, r, s)$ the mediane of $(m, r, s)$.

Proposition A.2. Let $u, v: \bar{M} \rightarrow \mathbb{C}$ be two dynamically Lipschitz continuous functions, with respect to $d_{\xi}$ with $\xi \in(0,1)$. Then

$$
\begin{aligned}
A_{0}(u, v) & =\mathbb{E}_{\bar{\mu}}[u] \cdot \mathbb{E}_{\bar{\mu}}[v] \\
A_{1}(u, v) & =i \lim _{n \rightarrow+\infty} \mathbb{E}_{\bar{\mu}}\left[u . S_{n} \cdot v \circ \bar{T}^{n}\right]=i B_{1}^{+}(u) \mathbb{E}_{\bar{\mu}}[v]+i B_{1}^{-}(v) \mathbb{E}_{\bar{\mu}}[u] \\
A_{2}(u, v) & =\lim _{n \rightarrow+\infty}\left(n \mathbb{E}_{\bar{\mu}}[u] \mathbb{E}_{\bar{\mu}}[v] \Sigma^{2}-\mathbb{E}_{\bar{\mu}}\left[u . S_{n}^{\otimes 2} \cdot v \circ \bar{T}^{n}\right]\right) \\
& =-2 B_{1}^{+}(u) \otimes B_{1}^{-}(v)-\mathbb{E}_{\bar{\mu}}[v] B_{2}^{+}(u)-\mathbb{E}_{\bar{\mu}}[u] B_{2}^{-}(v)+\mathbb{E}_{\bar{\mu}}[u] \mathbb{E}_{\bar{\mu}}[v] B_{0},
\end{aligned}
$$

Moreover

$$
\begin{aligned}
A_{3}(u, v)= & \lim _{n \rightarrow+\infty}\left(3 i n \Sigma^{2} \otimes \mathbb{E}_{\bar{\mu}}\left[u . S_{n} . v \circ \bar{T}^{n}\right]-i \mathbb{E}_{\bar{\mu}}\left[u . S_{n}^{\otimes 3} . v \circ \bar{T}^{n}\right]\right) \\
= & 3 A_{1}(u, v) \otimes B_{0}+3 i \Sigma^{2} \otimes\left(\mathbb{E}_{\bar{\mu}}[u] B_{0}^{-}(v)+\mathbb{E}_{\bar{\mu}}[v] B_{0}^{+}(u)\right) \\
& -i \mathbb{E}_{\bar{\mu}}[v] B_{3}^{+}(u)-i \mathbb{E}_{\bar{\mu}}[u] B_{3}^{-}(v)-3 i B_{2}^{-}(v) \otimes B_{1}^{+}(u)-3 i B_{2}^{+}(u) \otimes B_{1}^{-}(v)
\end{aligned}
$$


and

$$
\begin{aligned}
A_{4}(u, v)= & \lim _{n \rightarrow+\infty} \mathbb{E}_{\bar{\mu}}\left[u . S_{n}^{\otimes 4} \cdot v \circ \bar{T}^{n}\right]+\left(\lambda^{-n}\right)_{0}^{(4)} \mathbb{E}_{\bar{\mu}}[u] \mathbb{E}_{\bar{\mu}}[v]+6 n \Sigma^{2} \otimes \mathbb{E}_{\bar{\mu}}\left[u . S_{n}^{\otimes 2} \cdot v \circ \bar{T}^{n}\right] \\
= & \left.6 B_{0} A_{2}(u, v)-6 \Sigma^{2} \otimes\left(\mathbb{E}_{\bar{\mu}}[u] B_{0,2}^{-}(v)\right)-6 \mathbb{E}_{\bar{\mu}}[v] B_{0,2}^{+}(u)\right) \\
& +\mathbb{E}_{\bar{\mu}}[u] \mathbb{E}_{\bar{\mu}}[v]\left(A_{4}(\mathbf{1}, \mathbf{1})-6 B_{0}^{\otimes 2}\right) \\
& -12 \Sigma^{2} \otimes\left(B_{1}^{+}(u) \otimes B_{0}^{-}(v)+B_{1}^{-}(v) \otimes B_{0}^{+}(u)-B_{1}^{+}(u) \otimes B_{1}^{-}(v)\right) \\
& +4 B_{1}^{+}(u) \otimes B_{3}^{-}(v)+6 B_{2}^{+}(u) \otimes B_{2}^{-}(v)+4 B_{1}^{-}(v) \otimes B_{3}^{+}(u) .
\end{aligned}
$$

Proof. As in the proof of Theorem 4.4, we set

$$
A_{m, n}(u, v):=\left(\mathbb{E}_{\bar{\mu}}\left[v \cdot e^{i t * S_{n}} \cdot u \circ \bar{T}^{n}\right] / \lambda_{t}^{n}\right)_{\mid t=0}^{(m)} .
$$

We will only use Proposition A.1 and the fact that $\lambda_{t}=1-\frac{1}{2} \Sigma^{2} * t^{\otimes 2}+\frac{1}{4 !} \lambda_{0}^{(4)} * t^{\otimes 4}+o\left(|t|^{4}\right)$ to compute $A_{m}(u, v)=\lim _{n \rightarrow+\infty} A_{m, n}(u, v)$.

- First we observe that $A_{0, n}(u, v)=\mathbb{E}_{\bar{\mu}}\left[u . v \circ \bar{T}^{n}\right]$ and we apply Proposition A.1.

- Second,

$$
\begin{aligned}
A_{1, n}(u, v) & =i \mathbb{E}_{\bar{\mu}}\left[u . S_{n} . v \circ \bar{T}^{n}\right]=i \sum_{k=0}^{n-1} \mathbb{E}_{\bar{\mu}}\left[u . \kappa \circ \bar{T}^{k} . v \circ \bar{T}^{n}\right] \\
& =i \sum_{k=0}^{\lfloor n / 2\rfloor} \mathbb{E}_{\bar{\mu}}\left[u . \kappa \circ \bar{T}^{k}\right] \mathbb{E}_{\bar{\mu}}[v]+i \sum_{\lfloor n / 2\rfloor+1}^{n-1} \mathbb{E}_{\bar{\mu}}[u] \mathbb{E}_{\bar{\mu}}\left[v . \kappa \circ \bar{T}^{-(n-k)}\right]+O\left(n \vartheta_{0}^{n / 2}\|u\|_{(\xi)}\|u\|_{(\xi)}\right) \\
& =i \mathbb{E}_{\bar{\mu}}[v] \sum_{k \geq 0} \mathbb{E}_{\bar{\mu}}\left[u . \kappa \circ \bar{T}^{k}\right]+i \mathbb{E}_{\bar{\mu}}[u] \sum_{m \leq-1} \mathbb{E}_{\bar{\mu}}\left[v . \kappa \circ \bar{T}^{m}\right]+O\left(n \vartheta_{0}^{n / 2}\|u\|_{(\xi)}\|u\|_{(\xi)}\right),
\end{aligned}
$$

where we used several times Proposition A.1, combined with the fact that $\mathbb{E}_{\bar{\mu}}[\kappa]=0$.

- Third,

$$
\begin{aligned}
A_{2, n}(u, v)= & -\mathbb{E}_{\bar{\mu}}\left[u \cdot S_{n}^{\otimes 2} \cdot v \circ \bar{T}^{n}\right]+n \Sigma^{2} \mathbb{E}_{\bar{\mu}}[u] \mathbb{E}_{\bar{\mu}}[v] \\
= & -\sum_{k, m=0}^{n-1} \mathbb{E}_{\bar{\mu}}\left[u \cdot\left(\kappa \circ \bar{T}^{k} \otimes \kappa \circ \bar{T}^{m}\right) \cdot v \circ \bar{T}^{n}\right]+n \Sigma^{2} \mathbb{E}_{\bar{\mu}}[u] \mathbb{E}_{\bar{\mu}}[v] \\
= & -\sum_{k, m=0}^{n-1} \mathbb{E}_{\bar{\mu}}\left[\tilde{u} \kappa \circ \bar{T}^{k} \otimes \kappa \circ \bar{T}^{m} \cdot \tilde{v} \circ \bar{T}^{n}\right] \\
& -\sum_{k, m=0}^{n-1}\left(\mathbb{E}_{\bar{\mu}}[u] \mathbb{E}_{\bar{\mu}}\left[\kappa \circ \bar{T}^{k} \otimes \kappa \circ \bar{T}^{m} \tilde{v} \circ \bar{T}^{n}\right]+\mathbb{E}_{\bar{\mu}}\left[\tilde{u} . \kappa \circ \bar{T}^{k} \otimes \kappa \circ \bar{T}^{m}\right] \mathbb{E}_{\bar{\mu}}[v]\right) \\
& \quad+\left(n \Sigma^{2}-\sum_{k, m=0}^{n-1} \mathbb{E}_{\bar{\mu}}\left[\kappa \circ \bar{T}^{k} \otimes \kappa \circ \bar{T}^{m}\right]\right) \mathbb{E}_{\bar{\mu}}[u] \mathbb{E}_{\bar{\mu}}[v]
\end{aligned}
$$

- On the first hand

$$
\begin{aligned}
n \Sigma^{2}-\sum_{k, m=0}^{n-1} \mathbb{E}_{\bar{\mu}}\left[\kappa \circ \bar{T}^{k} \otimes \kappa \circ \bar{T}^{m}\right] & =n \sum_{k \in \mathbb{Z}} \mathbb{E}_{\bar{\mu}}\left[\kappa \otimes \kappa \circ \bar{T}^{k}\right]-\sum_{k=-n}^{n}(n-|k|) \mathbb{E}_{\bar{\mu}}\left[\kappa \otimes \kappa \circ \bar{T}^{k}\right] \\
& =\sum_{k \in \mathbb{Z}} \min (n,|k|) \mathbb{E}_{\bar{\mu}}\left[\kappa \otimes \kappa \circ \bar{T}^{k}\right],
\end{aligned}
$$

which converges to $\sum_{k \in \mathbb{Z}}|k| \mathbb{E}_{\bar{\mu}}\left[\kappa \otimes \kappa \circ \bar{T}^{k}\right]$. 
- On the second hand, for $0 \leq k \leq m \leq n$, due to Proposition A.1 (treating separately the cases $k \geq n / 3, m-n \geq n / 3$ et $n-m \geq n / 3)$,

$\mathbb{E}_{\bar{\mu}}\left[\tilde{u} . \kappa \circ \bar{T}^{k} \otimes \kappa \circ \bar{T}^{m} . \tilde{v} \circ \bar{T}^{n}\right]=\mathbb{E}_{\bar{\mu}}\left[\tilde{u} . \kappa \circ \bar{T}^{k}\right] \otimes \mathbb{E}_{\bar{\mu}}\left[\tilde{v} . \kappa \circ \bar{T}^{n-m}\right]+O\left(\|u\|_{(\xi)}\|v\|_{(\xi)} \vartheta_{0}^{n / 3}\right)$.

Analogously

Hence

$$
\begin{gathered}
\mathbb{E}_{\bar{\mu}}\left[\kappa \circ \bar{T}^{k} \otimes \kappa \circ \bar{T}^{m} \tilde{v} \circ \bar{T}^{n}\right]=O\left(\|v\|_{(\xi)} \vartheta_{0}^{(n-k) / 2}\right) \\
\mathbb{E}_{\bar{\mu}}\left[\tilde{u} . \kappa \circ \bar{T}^{k} \otimes \kappa \circ \bar{T}^{m}\right]=O\left(\|u\|_{(\xi)} \vartheta_{0}^{m / 2}\right) .
\end{gathered}
$$

$$
\begin{aligned}
& \sum_{k, m=0}^{n-1} \mathbb{E}_{\bar{\mu}}\left[\tilde{u} . \kappa \circ \bar{T}^{k} \otimes \kappa \circ \bar{T}^{m}\right]=B_{2}^{+}(\tilde{u})+O\left(\vartheta_{0}^{n / 2}\|u\|_{(\xi)}\right), \\
& \sum_{k, m=0}^{n-1} \mathbb{E}_{\bar{\mu}}\left[\kappa \circ \bar{T}^{k} \otimes \hat{\kappa} \circ \bar{T}^{m} \tilde{v} \circ \bar{T}^{n}\right]=B_{2}^{-}(v)+O\left(\vartheta_{0}^{n / 2}\|v\|_{(\xi)},\right.
\end{aligned}
$$

and

$$
\begin{aligned}
& \sum_{k, m=0}^{n-1} \mathbb{E}_{\bar{\mu}}\left[\tilde{u} . \kappa \circ \bar{T}^{k} \otimes \kappa \circ \bar{T}^{m} . \tilde{v} \circ \bar{T}^{n}\right] \\
= & \left(\sum_{k=0}^{n-1} \mathbb{E}_{\bar{\mu}}\left[\tilde{u} . \kappa^{\otimes 2} \circ \bar{T}^{k} . \tilde{v} \circ \bar{T}^{n}\right]+2 \sum_{0 \leq k<m<n} \mathbb{E}_{\bar{\mu}}\left[\tilde{u} . \kappa \circ \bar{T}^{k} \otimes \kappa \circ \bar{T}^{m} . \tilde{v} \circ \bar{T}^{n}\right]\right) \\
= & 2 \sum_{0 \leq k<m<n} \mathbb{E}_{\bar{\mu}}\left[\tilde{u} .\left(\kappa \circ \bar{T}^{k}\right)\right] \otimes \mathbb{E}_{\hat{\mu}}\left[\tilde{v} . \bar{\kappa} \circ \bar{T}^{n-m}\right]+O\left(\vartheta_{0}^{n / 2}\|u\|_{(\xi)}\|v\|_{(\xi)}\right) \\
= & 2 B_{1}^{+}(u) \otimes B_{1}^{-}(v)+O\left(\vartheta_{0}^{n / 2}\|u\|_{(\xi)}\|v\|_{(\xi)}\right),
\end{aligned}
$$

where we used the fact that $\mathbb{E}_{\bar{\mu}}\left[\tilde{u} . \kappa^{\otimes 2} \circ \bar{T}^{k} . \tilde{v} \circ \bar{T}^{n}\right]=O\left(\|u\|_{(\xi)}\|v\|_{(\xi)} \vartheta_{0}^{n / 2}\right)$.

Therefore we have proved (42).

- Let us prove (43). By bilinearity, we have

$$
A_{3, n}(u, v)=A_{3, n}(\tilde{u}, \tilde{v})+\mathbb{E}_{\bar{\mu}}[u] A_{3, n}(\mathbf{1}, \tilde{v})+\mathbb{E}_{\bar{\mu}}[v] A_{3, n}(\tilde{u}, \mathbf{1})+\mathbb{E}_{\bar{\mu}}[u] \mathbb{E}_{\bar{\mu}}[v] A_{3, n}(\mathbf{1}, \mathbf{1}) .
$$

Note that

$$
A_{3, n}(\mathbf{1}, \mathbf{1})=-i \mathbb{E}_{\bar{\mu}}\left[S_{n}^{\otimes 3}\right]=0 .
$$

since $\left(S_{n}\right)_{n}$ has the same distribution as $\left(-S_{n}\right)_{n}$ (see the begining of the proof of Proposition 3.1). We will use the following notations: $c_{(k, m, r)}$ denotes the number of uples made of $k, m, r$ (with their multiplicities) and we will write $\overbrace{F}$ for $F-\mathbb{E}_{\bar{\mu}}[F]$ when $F$ is given by a long formula.

- We start with the study of $A_{3, n}(\tilde{u}, \mathbf{1})$.

$$
\begin{aligned}
A_{3, n}(\tilde{u}, \mathbf{1})= & -i \mathbb{E}_{\bar{\mu}}\left[\tilde{u} . S_{n}^{\otimes 3}\right]+3 i n \Sigma^{2} \otimes \mathbb{E}_{\bar{\mu}}\left[\tilde{u} . S_{n}\right] \\
= & -i \sum_{0 \leq k \leq m \leq r \leq n-1} c_{k, m, r} \mathbb{E}_{\bar{\mu}}\left[\tilde{u} . \kappa \circ \bar{T}^{k} \otimes \kappa \circ \bar{T}^{m} \otimes \kappa \circ \bar{T}^{r}\right]+3 i n \Sigma^{2} \otimes \mathbb{E}_{\bar{\mu}}\left[\tilde{u} . S_{n}\right] \\
= & -i \sum_{0 \leq k \leq m \leq r \leq n-1} c_{k, m, r} \mathbb{E}_{\bar{\mu}}\left[\tilde{u} . \kappa \circ \bar{T}^{k}\right] \otimes \mathbb{E}_{\bar{\mu}}\left[\kappa \circ \bar{T}^{m} \otimes \kappa \circ \bar{T}^{r}\right]+3 i n \Sigma^{2} \otimes \mathbb{E}_{\bar{\mu}}\left[\tilde{u} . S_{n}\right] \\
& -i \sum_{0 \leq k \leq m \leq r \leq n-1} c_{k, m, r} \mathbb{E}_{\bar{\mu}}[\overbrace{\tilde{u} . \kappa \circ \bar{T}^{k}}^{\tau_{\kappa \circ \bar{T}^{m} \otimes \kappa \circ \bar{T}^{r}}}]
\end{aligned}
$$




$$
\begin{aligned}
& A_{3, n}(\tilde{u}, \mathbf{1}) \\
& =-3 i \sum_{k \geq 0} \sum_{m \in \mathbb{Z}} \max (0, n-|m|-k) \mathbb{E}_{\bar{\mu}}\left[\tilde{u} . \kappa \circ \bar{T}^{k}\right] \otimes \mathbb{E}_{\bar{\mu}}\left[\kappa . \kappa \circ \bar{T}^{m}\right]+3 i n \Sigma^{2} \otimes \mathbb{E}_{\bar{\mu}}\left[\tilde{u} . S_{n}\right] \\
& -i \sum_{k, m, r=0}^{n-1} \mathbb{E}_{\bar{\mu}}[\overbrace{\tilde{u} . \kappa \circ \bar{T}^{\min (k, m, r)}}^{\tau_{\kappa \circ \bar{T}^{\operatorname{med}(k, m, r)} \otimes \kappa \circ \bar{T}^{\max (k, m, r)}}}] \\
& =3 i \sum_{k \geq 0} \sum_{m \in \mathbb{Z}}(|m|+k) \mathbb{E}_{\bar{\mu}}\left[\tilde{u} . \kappa \circ \bar{T}^{k}\right] \otimes \mathbb{E}_{\bar{\mu}}\left[\kappa . \kappa \circ \bar{T}^{m}\right] \\
& -3 i n\left(B_{1}^{+}(u)-\mathbb{E}_{\bar{\mu}}\left[\tilde{u} . S_{n}\right]\right) \otimes \Sigma^{2}-i B_{3}^{+}(\tilde{u})+O\left(\vartheta_{0}^{n / 3}\|u\|_{(\xi)}\right)
\end{aligned}
$$

and so

$$
A_{3, n}(\tilde{u}, \mathbf{1})=-i B_{3}^{+}(\tilde{u})+3 i B_{0}^{+}(\tilde{u}) \otimes \Sigma^{2}+3 i B_{0} \otimes B_{1}^{+}(\tilde{u}) .
$$

- Analogously,

$$
A_{3, n}(\mathbf{1}, \tilde{v})=-i B_{3}^{-}(\tilde{v})+3 i B_{0}^{-}(\tilde{v}) \otimes \Sigma^{2}+3 i B_{0} \otimes B_{1}^{-}(\tilde{v}) .
$$

- Finally

$$
\begin{aligned}
A_{3, n}(\tilde{u}, \tilde{v}) & =-i \mathbb{E}_{\bar{\mu}}\left[\tilde{u} . S_{n}^{\otimes 3} . \tilde{v} \circ \bar{T}^{n}\right]+3 i n \Sigma^{2} \otimes \mathbb{E}_{\bar{\mu}}\left[\tilde{u} . S_{n} . \tilde{v} \circ \bar{T}^{n}\right] \\
& =-i \sum_{k, m, r=0}^{n-1} \mathbb{E}_{\bar{\mu}}\left[\tilde{u} . \kappa \circ \bar{T}^{k} \otimes \kappa \circ \bar{T}^{m} \otimes \kappa \circ \bar{T}^{r} . \tilde{v} \circ \bar{T}^{n}\right]+3 i n \Sigma^{2} \otimes \tilde{A}_{1, n}(\tilde{u}, \tilde{v}) \\
& =-i \sum_{k, m, r=0}^{n-1} \mathbb{E}_{\bar{\mu}}\left[\tilde{u} . \kappa \circ \bar{T}^{k} \otimes \kappa \circ \bar{T}^{m} \otimes \kappa \circ \bar{T}^{r} . \tilde{v} \circ \bar{T}^{n}\right]+O\left(n^{2} \vartheta_{0}^{n / 2}\|u\|_{(\xi)}\|v\|_{(\xi)}\right) .
\end{aligned}
$$

Assume $0 \leq k \leq m \leq r \leq n-1$. Considering separately the cases $k \geq n / 4$, $m-k \geq n / 4, r-m \geq n / 4$ and $n-r \geq n / 4$, we observe that

$$
\begin{aligned}
& \mathbb{E}_{\bar{\mu}}\left[\tilde{u} . \kappa \circ \hat{T}^{k} \otimes \kappa \circ \bar{T}^{m} . \otimes \kappa \circ \bar{T}^{r} . \tilde{v} \circ \bar{T}^{n}\right] \\
& =\mathbb{E}_{\bar{\mu}}\left[\tilde{u} . \kappa \circ \bar{T}^{k}\right] \otimes \mathbb{E}_{\bar{\mu}}\left[\tilde{v} . \kappa \circ \bar{T}^{-(n-r)} \otimes \kappa \circ \bar{T}^{-(n-m)}\right] \\
& +\mathbb{E}_{\bar{\mu}}\left[\tilde{v} . \kappa \circ \bar{T}^{-(n-r)}\right] \otimes \mathbb{E}_{\bar{\mu}}\left[\tilde{u} . \kappa \circ \bar{T}^{k} \otimes \kappa \circ \bar{T}^{m}\right]+O\left(\vartheta_{0}^{n / 4}\|v\|_{(\xi)}\|u\|_{(\xi)}\right) .
\end{aligned}
$$

And so

$$
A_{3, n}(\tilde{u}, \tilde{v})=-3 i B_{1}^{+}(\tilde{u}) B_{2}^{-}(\tilde{v})-3 i B_{1}^{-}(\tilde{v}) B_{2}^{+}(\tilde{u}) .
$$

This combined with (50), (53) and (54) leads to (43).

- It remains to prove (44). Observe first that

$$
\begin{aligned}
A_{4, n}(u, v) & =\left(\lambda^{-n}\right)_{0}^{(4)} \mathbb{E}_{\bar{\mu}}[\bar{u}] \mathbb{E}_{\bar{\mu}}[\bar{v}]+6 n \Sigma^{2} \otimes \mathbb{E}_{\bar{\mu}}\left[u . S_{n}^{\otimes 2} \cdot v \circ \bar{T}^{n}\right]+\mathbb{E}_{\bar{\mu}}\left[u . S_{n}^{\otimes 4} \cdot v \circ \bar{T}^{n}\right] \\
& =\left(\lambda^{-n}\right)_{0}^{(4)} \mathbb{E}_{\bar{\mu}}[\bar{u}] \mathbb{E}_{\bar{\mu}}[\bar{v}]+6 n \Sigma^{2} \otimes\left(n \Sigma^{2} \mathbb{E}_{\bar{\mu}}[u] \mathbb{E}_{\bar{\mu}}[v]-A_{2, n}(u, v)\right)+\mathbb{E}_{\bar{\mu}}\left[u . S_{n}^{\otimes 4} \cdot v \circ \bar{T} 5 \eta \dagger\right)
\end{aligned}
$$

where we used (44). Note that

$$
\begin{aligned}
\mathbb{E}_{\bar{\mu}}\left[u . S_{n}^{\otimes 4} . v \circ \bar{T}^{n}\right]= & \mathbb{E}_{\bar{\mu}}\left[\tilde{u} . S_{n}^{\otimes 4} . \tilde{v} \circ \bar{T}^{n}\right]+\mathbb{E}_{\bar{\mu}}[u] \mathbb{E}_{\bar{\mu}}\left[S_{n}^{\otimes 4} . \tilde{v} \circ \bar{T}^{n}\right] \\
& +\mathbb{E}_{\bar{\mu}}[v] \mathbb{E}_{\bar{\mu}}\left[\tilde{u} \cdot S_{n}^{\otimes 4}\right]+\mathbb{E}_{\bar{\mu}}[u] \mathbb{E}_{\bar{\mu}}[v] \mathbb{E}_{\bar{\mu}}\left[S_{n}^{\otimes 4}\right] .
\end{aligned}
$$

We now study separately each term of the right hand side of this last formula. 
- First:

$$
\begin{aligned}
& \mathbb{E}_{\bar{\mu}}\left[\tilde{u} . S_{n}^{\otimes 4} . \tilde{v} \circ \bar{T}^{n}\right] \\
& =\sum_{k, m, r, s=0}^{n-1} \mathbb{E}_{\hat{\mu}}\left[\tilde{u} . \kappa \circ \bar{T}^{k} \otimes \kappa \circ \bar{T}^{m} . \kappa \circ \bar{T}^{r} \otimes \kappa \circ \bar{T}^{s} . \tilde{v} \circ \bar{T}^{n}\right] \\
& =\sum_{0 \leq k \leq m \leq r \leq s \leq n-1} c_{(k, m, r, s)} \mathbb{E}_{\bar{\mu}}[\tilde{u} . \kappa \circ \bar{T}^{k} \otimes \overbrace{\kappa \otimes \kappa \circ \bar{T}^{r-m}}^{\tau_{\kappa}} \bar{T}^{m} . \kappa \circ \bar{T}^{s} . \tilde{v} \circ \bar{T}^{n}] \\
& +\sum_{0 \leq k \leq m \leq r \leq s \leq n-1} c_{(k, m, r, s)} \mathbb{E}_{\bar{\mu}}\left[\tilde{u} . \kappa \circ \bar{T}^{k} . \kappa \circ \bar{T}^{s} . \tilde{v} \circ \bar{T}^{n}\right] \otimes \mathbb{E}_{\bar{\mu}}\left[\kappa \otimes \kappa \circ \bar{T}^{r-m}\right]
\end{aligned}
$$

with $c_{(k, m, r, s)}$ the number of 4-uples made of $k, m, r, s$ (with the same multiplicities). Due to (46),

$$
\begin{aligned}
& \sum_{0 \leq k \leq m \leq r \leq s \leq n-1} c_{(k, m, r, s)} \mathbb{E}_{\bar{\mu}}\left[\tilde{u} . \kappa \circ \bar{T}^{k} \otimes \kappa \circ \bar{T}^{s} . \tilde{v} \circ \bar{T}^{n}\right] \otimes \mathbb{E}_{\bar{\mu}}\left[\kappa \otimes \kappa \circ \bar{T}^{r-m}\right] \\
& =\sum_{0 \leq k \leq m \leq r \leq s \leq n-1} c_{(k, m, r, s)} \mathbb{E}_{\bar{\mu}}\left[\tilde{u} . \kappa \circ \bar{T}^{k}\right] \otimes \mathbb{E}_{\bar{\mu}}\left[\tilde{v} . \kappa \circ \bar{T}^{-(n-s)}\right] \otimes \mathbb{E}_{\bar{\mu}}\left[\kappa \otimes \kappa \circ \bar{T}^{r-m}\right]+O\left(n^{4} \vartheta_{0}^{n / 3}\|u\|_{(\xi)}\|v\|_{(\xi)}\right) \\
& =\sum_{k \geq 0} \mathbb{E}_{\bar{\mu}}\left[\tilde{u} . \kappa \circ \bar{T}^{k}\right] \otimes \sum_{s \geq 1} \mathbb{E}_{\bar{\mu}}\left[\tilde{v} . \kappa \circ \bar{T}^{-s}\right] \otimes \sum_{m=k}^{n-s} \sum_{r=m}^{n-s} c_{(k, m, r, n-s)} \mathbb{E}_{\bar{\mu}}\left[\kappa \otimes \kappa \circ \bar{T}^{r-m}\right]+O\left(n^{4} \vartheta_{0}^{n / 3}\|u\|_{(\xi)}\|v\|_{(\xi)}\right) \\
& =\sum_{k \geq 0} \mathbb{E}_{\bar{\mu}}\left[\tilde{u} . \kappa \circ \bar{T}^{k}\right] \otimes \sum_{s \geq 1} \mathbb{E}_{\bar{\mu}}\left[\tilde{v} . \kappa \circ \bar{T}^{-s}\right] \otimes 12 \mathbb{E}_{\bar{\mu}}\left[S_{n-s-k+1}^{\otimes 2}\right]+O\left(n^{4} \vartheta_{0}^{n / 3}\|u\|_{(\xi)}\|v\|_{(\xi)}\right) \\
& =\sum_{k \geq 0} \mathbb{E}_{\bar{\mu}}\left[\tilde{u} . \kappa \circ \hat{T}^{k}\right] \otimes \sum_{s \geq 1} \mathbb{E}_{\bar{\mu}}\left[\tilde{v} . \kappa \circ \bar{T}^{-s}\right] 12\left((n-s-k+1) \Sigma^{2}-\sum_{r \in \mathbb{Z}}|r| \mathbb{E}_{\hat{\mu}}\left[\hat{\kappa} \otimes \hat{\kappa} \circ \hat{T}^{r}\right]+O\left(n^{4} \vartheta_{0}^{n / 3}\|u\|_{(\xi)}\|v\|_{(\xi)}\right.\right. \\
& =12 B_{1}^{+}(\tilde{u}) B_{1}^{-}(\tilde{v})\left(n \Sigma^{2}-\sum_{r \in \mathbb{Z}}|r| \mathbb{E}_{\hat{\mu}}\left[\hat{\kappa} \otimes \hat{\kappa} \circ \hat{T}^{r}\right]\right) \\
& -12 \sum_{k \geq 0} \mathbb{E}_{\bar{\mu}}\left[\tilde{u} . \kappa \circ \bar{T}^{k}\right] \otimes \sum_{s \geq 1} \mathbb{E}_{\bar{\mu}}\left[\tilde{v} . \kappa \circ \bar{T}^{-s}\right](s+k-1) \otimes \Sigma^{2}+O\left(n^{4} \vartheta_{0}^{n / 3}\|u\|_{(\xi)}\|v\|_{(\xi)}\right) .
\end{aligned}
$$

But, on the other hand, treating separately the cases $k \geq n / 5, m-k \geq n / 5, r-m \geq$ $n / 5, s-r \geq n / 5$ and $n-s \geq n / 5$, we obtain that, for every $0 \leq k \leq m \leq r \leq s \leq n$,

$\mathbb{E}_{\bar{\mu}}[\tilde{u} . \kappa \circ \bar{T}^{k} \otimes \overbrace{\kappa \otimes \kappa \circ \bar{T}^{r-m}}^{\tau} \circ \bar{T}^{m} \otimes \kappa \circ \bar{T}^{s} . \tilde{v} \circ \bar{T}^{n}]$

$=\mathbb{E}_{\bar{\mu}}\left[\tilde{u} . \kappa \circ \bar{T}^{k}\right] \otimes \mathbb{E}_{\bar{\mu}}[\overbrace{\kappa \otimes \kappa \circ \bar{T}^{r-m}}^{2} \bar{T}^{m} \otimes \kappa \circ \bar{T}^{s} . \tilde{v} \circ \bar{T}^{n}]$

$+\mathbb{E}_{\bar{\mu}}\left[\tilde{u} . \kappa \circ \bar{T}^{k} \otimes \hat{\kappa} \circ \hat{T}^{m}\right] \otimes \mathbb{E}_{\bar{\mu}}\left[\kappa \circ \bar{T}^{r} \otimes \kappa \circ \bar{T}^{s} . \tilde{v} \circ \bar{T}^{n}\right]$

$+\mathbb{E}_{\bar{\mu}}[\tilde{u} . \kappa \circ \bar{T}^{k} \otimes \overbrace{\kappa \otimes \kappa \circ \bar{T}^{r-m}} \circ \bar{T}^{m}] \otimes \mathbb{E}_{\bar{\mu}}\left[\kappa \circ \bar{T}^{s} . \tilde{v} \circ \bar{T}^{n}\right]+O\left(\vartheta_{0}^{n / 5}\|u\|_{(\xi)}\|v\|_{(\xi)}\right)$.

Due to (48),

$$
\mathbb{E}_{\bar{\mu}}[\overbrace{\kappa . \kappa \circ \bar{T}^{r-m}}^{\overbrace{T}} \bar{T}^{m} . \kappa \circ \bar{T}^{s} . \tilde{v} \circ \bar{T}^{n}]=O\left(\vartheta_{0}^{n-m}\|u\|_{(\xi)}\|v\|_{(\xi)}\right),
$$




$$
\begin{aligned}
& \mathbb{E}_{\bar{\mu}}[\tilde{u} . \kappa \circ \bar{T}^{k} . \overbrace{\kappa . \kappa \circ \bar{T}^{r-m}}^{\tau_{T}} \bar{T}^{m}] \mathbb{E}_{\bar{\mu}}\left[\kappa \circ \bar{T}^{s} . \tilde{v} \circ \bar{T}^{n}\right]=O\left(\vartheta_{0}^{m} \vartheta_{0}^{n-s}\|u\|_{(\xi)}\|v\|_{(\xi)}\right), \\
& \mathbb{E}_{\bar{\mu}}\left[\tilde{u} . \kappa \circ \bar{T}^{k} \otimes \kappa \circ \bar{T}^{m}\right]=O\left(\vartheta_{0}^{m}\|u\|_{(\xi)}\right) .
\end{aligned}
$$

Therefore

$$
\begin{aligned}
& \sum_{0 \leq k \leq m \leq r \leq s \leq n-1} c_{(k, m, r, s)} \mathbb{E}_{\bar{\mu}}[\tilde{u} . \kappa \circ \bar{T}^{k} \otimes \overbrace{\kappa \circ \bar{T}^{m} \otimes \kappa \circ \bar{T}^{m}}^{\widetilde{ }} \otimes \circ \bar{T}^{s} . \tilde{v} \circ \bar{T}^{n}] \\
& =4 \sum_{k \geq 0} \mathbb{E}_{\bar{\mu}}\left[\tilde{u} . \kappa \circ \bar{T}^{k}\right] B_{3}^{-}(\tilde{v})+4 B_{3}^{+}(\tilde{u}) \otimes \sum_{s \geq 1} \mathbb{E}_{\bar{\mu}}\left[\kappa \circ \bar{T}^{-s} . \tilde{v}\right] \\
& +6 \sum_{m, k \geq 0} \mathbb{E}_{\bar{\mu}}\left[\tilde{u} . \kappa \circ \bar{T}^{k} \otimes \kappa \circ \bar{T}^{m}\right] \otimes \sum_{r, s \geq 1} \mathbb{E}\left[\tilde{v} . \kappa \circ \bar{T}^{-r} \otimes \kappa \circ \bar{T}^{-s}\right] \\
& +O\left(\vartheta_{0}^{n / 5}\|u\|_{(\xi)}\|v\|_{(\xi)}\right) .
\end{aligned}
$$

Putting together (57), (59), (60) and (62) leads to

$$
\begin{aligned}
A_{4, n}(\tilde{u}, \tilde{v})= & -12 \sum_{k \geq 0} \mathbb{E}_{\hat{\mu}}\left[\tilde{u} . \kappa \circ \hat{T}^{k}\right] \sum_{s \geq 1} \mathbb{E}_{\hat{\mu}}\left[\kappa \circ \bar{T}^{-s} . \tilde{v}\right](s+k-1) \otimes \Sigma^{2} \\
& +4 B_{1}^{+}(u) \otimes B_{3}^{-}(\tilde{v})+4 B_{1}^{-}(v) \otimes B_{3}^{+}(\tilde{u}) \\
& +6 B_{2}^{+}(u) \otimes B_{2}^{-}(v)-12 B_{1}^{+}(\tilde{u}) \otimes B_{1}^{-}(\tilde{v}) \otimes B_{0}+O\left(\vartheta_{0}^{n / 5}\|u\|_{(\xi)}\|v\|_{(\xi)}\right)
\end{aligned}
$$

- Second:

$$
\mathbb{E}_{\bar{\mu}}\left[\tilde{u} . S_{n}^{\otimes 4}\right]=\sum_{0 \leq k \leq m \leq r \leq s \leq n-1} c_{(k, m, r, s)} \mathbb{E}_{\bar{\mu}}\left[\tilde{u} . \kappa \circ \bar{T}^{k} \otimes \kappa \circ \bar{T}^{m} \otimes \kappa \circ \bar{T}^{r} \otimes \kappa \circ \bar{T}^{s}\right] .
$$

But, due to (55), for $0 \leq k \leq m \leq r \leq s \leq n-1$, we have

$$
\begin{aligned}
& \mathbb{E}_{\bar{\mu}}\left[\tilde{u} . \kappa \circ \bar{T}^{k} \otimes \kappa \circ \bar{T}^{m} \otimes \kappa \circ \bar{T}^{r} \otimes \kappa \circ \bar{T}^{s}\right] \\
& =\mathbb{E}_{\bar{\mu}}\left[\tilde{u} . \kappa \circ \bar{T}^{k}\right] \otimes \mathbb{E}_{\bar{\mu}}\left[\kappa \otimes \kappa \circ \bar{T}^{r-m} \otimes \kappa \circ \bar{T}^{s-m}\right] \\
& +\mathbb{E}_{\bar{\mu}}\left[\tilde{u} . \kappa \circ \bar{T}^{k} \otimes \kappa \circ \bar{T}^{m}\right] \otimes \mathbb{E}_{\bar{\mu}}\left[\kappa \otimes \kappa \circ \bar{T}^{s-r}\right]+O\left(\|u\|_{(\xi)}\|v\|_{(\xi)} \vartheta_{0}^{s / 3}\right) .
\end{aligned}
$$

Therefore

$$
\begin{aligned}
& \mathbb{E}_{\bar{\mu}}\left[\tilde{u} . S_{n}^{\otimes 4}\right]=4 \sum_{k \geq 0} \mathbb{E}_{\bar{\mu}}\left[S_{n-k}^{\otimes 3}\right] \\
& +6 \sum_{k, m \geq 0} \sum_{r \in \mathbb{Z}} \max (0,(n-\max (k, m)-|r|)) \mathbb{E}_{\bar{\mu}}\left[\tilde{u} . \kappa \circ \bar{T}^{k} \otimes \kappa \circ \bar{T}^{m}\right] \otimes \mathbb{E}_{\bar{\mu}}\left[\kappa \otimes \kappa \circ \bar{T}^{r}\right] \\
= & 6 n B_{2}^{+}(\tilde{u}) \otimes \Sigma^{2}-6 \sum_{k, m \geq 0} \sum_{r \in \mathbb{Z}}(\max (k, m)+|r|) \mathbb{E}_{\bar{\mu}}\left[\tilde{u} . \kappa \circ \bar{T}^{k} \otimes \kappa \circ \bar{T}^{m}\right] \otimes \mathbb{E}_{\bar{\mu}}\left[\kappa \otimes \kappa \circ \bar{T}^{r}\right]
\end{aligned}
$$$$
\text { since } \mathbb{E}_{\bar{\mu}}\left[S_{n}^{\otimes 3}\right]=0 \text {. It comes }
$$$$
\mathbb{E}_{\bar{\mu}}\left[\tilde{u} . S_{n}^{\otimes 4}\right]=6 n B_{2}^{+}(\tilde{u}) \otimes \Sigma^{2}-6 B_{0,2}^{+}(\tilde{u}) \otimes \Sigma^{2}-6 B_{2}^{+}(\tilde{u}) \otimes B_{0}+O\left(\vartheta_{0}^{n / 2}\right)
$$

- Analogously,

$$
\mathbb{E}_{\bar{\mu}}\left[\tilde{v} \circ \bar{T}^{n} \cdot S_{n}^{\otimes 4}\right]=6 n B_{2}^{-}(\tilde{v}) \otimes \Sigma^{2}-6 B_{0,2}^{-}(\tilde{v}) \otimes \Sigma^{2}-6 B_{2}^{-}(\tilde{v}) \otimes B_{0}+O\left(\vartheta_{0}^{n / 2}\right) .
$$

Formula (44) follows from (58), (63), (65) and (66). 
Proposition A.3. The fourth derivatives of $\lambda$ at 0 are given by

$$
\lambda_{0}^{(4)}=\lim _{n \rightarrow+\infty} \frac{\mathbb{E}_{\bar{\mu}}\left[S_{n}^{\otimes 4}\right]-3 n^{2}\left(\Sigma^{2}\right)^{\otimes 2}}{n}+3\left(\Sigma^{2}\right)^{\otimes 2}+6 \Sigma^{2} \otimes B_{0} .
$$

Proof. Derivating four times $\mathbb{E}_{\bar{\mu}}\left[e^{i t * S_{n}}\right]=\lambda_{t}^{n} \mathbb{E}_{\bar{\mu}}\left[e^{i t * S_{n}} / \lambda_{t}^{n}\right]$ leads to

$$
\begin{aligned}
\mathbb{E}_{\bar{\mu}}\left[S_{n}^{\otimes 4}\right] & =\left(\lambda^{n}\right)_{0}^{(4)}+6\left(\lambda^{n}\right)_{0}^{(2)} \otimes A_{2, n}(\mathbf{1}, \mathbf{1})+A_{4, n}(\mathbf{1}, \mathbf{1}) \\
& =n \lambda_{0}^{(4)}+3 n(n-1)\left(\lambda_{0}^{(2)}\right)^{\otimes 2}+6 n \lambda_{0}^{(2)} \otimes A_{2, n}(\mathbf{1}, \mathbf{1})+A_{4, n}(\mathbf{1}, \mathbf{1}),
\end{aligned}
$$

and we conclude due to (34) and due to $\lambda_{0}^{(2)}=-\Sigma^{2}$ (coming from Item (iii) of Proposition 4.11).

Acknowledgment. The author wishes to thank Damien Thomine for interesting discussions having led to an improvement of the assumption for the mixing result in the infinite horizon billiard case.

\section{REFERENCES}

[1] L. A. Bunimovich, N. I. Chernov \& Ya. G. Sinai, Markov partitions for two-dimensional hyperbolic billiards, Russ. Math. Survey 45 (1990), no 3, 105-152.

[2] L. A. Bunimovich, Ya. G. Sinai \& N. I. Chernov, Statistical properties of two-dimensional hyperbolic billiards. (Russian) Uspekhi Mat. Nauk 46 (1991), no. 4(280), 43-92, 192; translation in Russian Math. Surveys 46 (1991), no. 4, 47-106

[3] L. A. Bunimovich \& Ya. G. Sinai, Markov partitions for dispersed billiards, Comm. Math. Phys. 78 (1980), $247-280$

[4] L.A. Bunimovich, \& Ya. G. Sinai, Statistical properties of Lorentz gas with periodic configuration of scatterers, Comm. Math. Phys. 78 (1980/81), no. 4, 479-497.

[5] H. Bruin \& D. Terhesiu, Upper and lower bounds for the correlation function via inducing with general return times, Ergod. Th. Dyn. Sys, DOI: http://dx.doi.org/10.1017/etds.2016.20

[6] N. Chernov, Advanced statistical properties of dispersing billiards, Journal of Statistical Physics, 122 (2006), 1061-1094.

[7] N. Chernov \& R. Markarian, Chaotic billiards, Mathematical Surveys and Monographs, 127. American Mathematical Society, Providence, RI, (2006) xii+316 pp.

[8] J.P. Conze, Sur un critère de récurrence en dimension 2 pour les marches stationnaires, applications, Erg. Th. E Dynam. Syst. 19 (1999) 1233-1245.

[9] D. Dolgopyat, D. Szász \& T. Varjú, Recurrence properties of planar Lorentz process, Duke Math. J. 142 (2008) 241-281.

[10] S. Gouëzel, Correlation asymptotics from large deviations in dynamical systems with infinite measure, Colloquium Mathematicum 125 (2011) 193-212.

[11] Y. Guivarc'h \& J. Hardy, Théorèmes limites pour une classe de chaînes de Markov et applications aux difféomorphismes d'Anosov, Annales Inst. H. Poincaré (B), Probabilité et Statistiques 24 (1988) 73-98.

[12] Keller G., Liverani C. Stability of the Spectrum for Transfer Operators. Ann. Scuola Norm. Sup. Pisa. CI. Sci. (4) Vol. XXVIII (1999) 141-152.

[13] U. Krengel \& L. Sucheston, On mixing in infinite measure spaces, Z. Wehrsch. u. v. Geb. 13 (1969), $150-164$.

[14] C. Liverani \& D. Terhesiu, Mixing for some non-uniformly hyperbolic systems, Annales Henri Poincaré $\mathbf{1 7}$ (2016) 179-226.

[15] I. Melbourne \& D. Terhesiu, Operator renewal theory and mixing rates for dynamical systems with infinite measure, Invent. Math. 1 (2012) 61-110.

[16] S. V. Nagaev, Some limit theorems for stationary Markov chains, Theor. Probab. Appl. 2 (1957) 378-406; translation from Teor. Veroyatn. Primen. 2 (1958) 389-416

[17] S. V. Nagaev, More exact statement of limit theorems for homogeneous Markov chains, Theor. Probab. Appl. 6 (1961) 62-81; translation from Teor. Veroyatn. Primen 6 (1961) 67-86

[18] F. Pène, Applications des propriétés stochastiques de billards dispersifs, Comptes Rendus de l'Académie des Sciences 330 (I) (2009), 1103-1106.

[19] F. Pène, Planar Lorentz process in a random scenery, Annales de l'Institut Henri Poincaré, Probabilités et Statistiques 45 (3) (2009) 818-839.

[20] F. Pène, Asymptotic of the number of obstacles visited by the planar Lorentz process, Discrete and Continuous Dynamical Systems (A) 24 (2) (2009), 567-588. 
[21] F. Pène, Mixing rate in infinite measure for $\mathbb{Z}^{d}$-extensions, application to the periodic Sinai billiard, preprint.

[22] F. Pène and B. Saussol, Back to balls in billiards, Communications in Mathematical Physics 293 (2010), 837-866.

[23] F. Pène and D. Thomine, Potential kernel, hitting probabilities and distributional asymptotics arXiv:1702.06625

[24] K. Schmidt, On joint recurrence, C. R. Acad. Sci. 327 (1998) 837-842.

[25] N. Simányi, Toward a proof of recurrence for the Lorentz process, Banach Center Publications, PWN, Warsaw 23 (1989) 265-276.

[26] Y. Sinai, Dynamical systems with elastic reflections, Russ. Math. Surv. 25, No.2 (1970) 137-189

[27] D. Szász and T. Varjú, Local limit theorem for the Lorentz process and its recurrence in the plane, Erg. Th. Dyn. Syst. 24, No.1 (2004) 257-278

[28] D. Szász and T. Varjú, Limit Laws and Recurrence for the Planar Lorentz Process with Infinite Horizon, J. Statist. Phys. 129 (2007) 59-80.

[29] M. Thaler, The asymptotics of the Perron Perron-Frobenius operator of a class of interval maps preserving infinite measures, Studia Math. 143 (2000) 103-119.

[30] L.-S. Young, Statistical properties of dynamical systems with some hyperbolicity, Ann. of Math. 147 (1998) 585-650.

1) Université de Brest, Laboratoire de Mathématiques de Bretagne Atlantique, CNRS UMR 6205, France, 2) Institut Universitaire de France, 3) Université de Bretagne Loire

E-mail address: francoise.pene@univ-brest.fr 